\title{
Genome-wide identification and characterization of WRKY gene family in Salix suchowensis
}

\author{
Changwei Bi ${ }^{1}$, Yiqing $\mathrm{Xu}^{1}{ }^{1}$, Qiaolin $\mathrm{e}^{1}{ }^{1}$, Tongming Yin ${ }^{2}$, Ning Ye ${ }^{\text {Corresp. } 1}$ \\ ${ }^{1}$ College of Information Science and Technology, Nanjing Forestry University, Nanjing, Jiangsu, China \\ 2 College of Forest Resources and Environment, Nanjing Forestry University, Nanjing, Jiangsu, China \\ Corresponding Author: Ning Ye \\ Email address: yening@njfu.edu.cn
}

WRKY proteins are the zinc finger transcription factors that were first identified in plants. They can specifically interact with the W-box, which can be found in the promoter region of a large number of plant target genes, to regulate the expressions of downstream target genes. They also participate in diverse physiological and growing processes in plants. Prior to this study, a plenty of WRKY genes have been identified and characterized in herbaceous species, but there is no large-scale study of WRKY genes in willow. With the whole genome sequencing of Salix suchowensis, we have the opportunity to conduct the genome-wide research for willow WRKY gene family. In this study, we identified 85 WRKY genes in the willow genome and renamed them from SSWRKY1 to SsWRKY85 on the basis of their specific distributions on chromosomes. Due to their diverse structural features, the 85 willow WRKY genes could be further classified into three main groups (group I - III), with five subgroups (Ila - Ile) in group II. With the multiple sequence alignment and the manual search, we found three variations of the WRKYGQK heptapeptide: WRKYGRK, WKKYGQK and WRKYGKK, and four variations of the normal zinc finger motif, which might execute some new biological functions. In addition, the SsWRKY genes from the same subgroup share the similar exon-intron structures and conserved motif domains. Further studies of SsWRKY genes revealed that segmental duplication events played a more prominent role in the expansion of SsWRKY genes. Distinct expression profiles of SsWRKY genes with RNA sequencing data revealed that diverse expression patterns among five tissues, including tender roots, young leaves, vegetative buds, non-lignified stems and barks. With the analyses of WRKY gene family in willow, it is not only beneficial to complete the functional and annotation information of WRKY genes family in woody plants, but also provide important references to investigate the expansion and evolution of this gene family in flowering plants. 


\section{Genome-wide identification and characterization of WRKY}

\section{2 gene family in Salix suchowensis}

3 Changwei $\mathrm{Bi}^{1}$, Yiqing $\mathrm{Xu}^{1}$, Yanshu $\mathrm{Qu}^{1}$, Qiaolin $\mathrm{Ye}^{1}$, Tongming $\mathrm{Yin}^{2}$, Ning $\mathrm{Ye}^{{ }^{*}}$

4 1. College of Information Science and Technology, Nanjing Forestry University, Nanjing,

5 Jiangsu, China

6 2. College of Forest Resources and Environment, Nanjing Forestry University, Nanjing, Jiangsu,

7 China

$8 *$ Corresponding author:

$9 \quad$ Ning $\mathrm{Ye}^{1}$

10 Nanjing Forestry University, 159 Longpan Road, Nanjing, 210037, China

11 Email address: yening@njfu.edu.cn 


\section{Abstract}

13 WRKY proteins are the zinc finger transcription factors that were first identified in plants. They

14 can specifically interact with the W-box, which can be found in the promoter region of a large number of plant target genes, to regulate the expressions of downstream target genes. They also participate in diverse physiological and growing processes in plants. Prior to this study, a plenty of WRKY genes have been identified and characterized in herbaceous species, but there is no large-scale study of WRKY genes in willow. With the whole genome sequencing of Salix suchowensis, we have the opportunity to conduct the genome-wide research for willow WRKY gene family. In this study, we identified 85 WRKY genes in the willow genome and renamed them from SsWRKY1 to SsWRKY85 on the basis of their specific distributions on chromosomes. Due to their diverse structural features, the 85 willow WRKY genes could be further classified into three main groups (group I - III), with five subgroups (IIa - IIe) in group II.

24 With the multiple sequence alignment and the manual search, we found three variations of the

25 WRKYGQK heptapeptide: WRKYGRK, WKKYGQK and WRKYGKK, and four variations of the normal zinc finger motif, which might execute some new biological functions. In addition, the SsWRKY genes from the same subgroup share the similar exon-intron structures and conserved motif domains. Further studies of SsWRKY genes revealed that segmental duplication events played a more prominent role in the expansion of SsWRKY genes. Distinct expression profiles of SsWRKY genes with RNA sequencing data revealed that diverse expression patterns among five tissues, including tender roots, young leaves, vegetative buds, non-lignified stems and barks. With the analyses of WRKY gene family in willow, it is not only beneficial to complete the

33 functional and annotation information of WRKY genes family in woody plants, but also provide

34 important references to investigate the expansion and evolution of this gene family in flowering 35 plants. 


\section{Introduction}

37 Plants form a series of adjustment mechanisms to adapt diverse environment stress in their long evolutionary processes. Among the numerous adjustment mechanisms, transcription factors play important roles (Jang et al. 2010). In plants, WRKY proteins constitute a large family of transcription factors, involving in various physiological and developmental processes (Eulgem 2000; Rushton et al. 2010). Since the first WRKY gene was cloned and characterized from sweet

42 potato (Ishiguro \& Nakamura 1994), many corresponding studies have been conducted rapidly,

43 such as Arabidopsis thaliana, desert legume (Retama raetam), cotton (Gossypium arboreum),

44 rice (Oryza sativa), Pinus monticola, barley (Hordeum vulgare), sunflower, cucumber (Cucumis

45 sativus), poplar (Populus trichocarpa), tomato (Solanum lycopersicum) and grapevine (Vitis

46 vinifera) (Ding et al. 2015; Eulgem 2000; Giacomelli et al. 2010; Guo et al. 2014; He et al. 2012;

47 Huang et al. 2012; Ling et al. 2011; Liu \& Ekramoddoullah 2009; Mangelsen et al. 2008; Pnueli

48 et al. 2002; Wu 2005).

49 The existence of either one or two highly conserved WRKY domains is the most vital structural

50 characteristic of WRKY gene. WRKY gene consists of about 60 amino acid residues with a

51 conserved WRKYGQK heptapeptide at its N-termini, and a zinc finger motif $\left(\mathrm{C}-\mathrm{X}_{4-5}-\mathrm{C}-\mathrm{X}_{22-23}-\mathrm{H}-\right.$

$52 \mathrm{X}_{1}-\mathrm{H}$ or $\left.\mathrm{C}-\mathrm{X}_{7}-\mathrm{C}-\mathrm{X}_{23}-\mathrm{H}-\mathrm{X}_{1}-\mathrm{C}\right)$ at the $\mathrm{C}$-terminal region. Previous functional studies indicated that

53 WRKY genes could specifically interact with the W-box ([C/T]TGAC[T/C]), the promoter

54 region of plant target genes, to adjust the expressions of downstream target genes (Ciolkowski et

55 al. 2008). Additionally, SURE (sugar responsive elements), another prominent cis-element that

56 can promote transcription processes, was also found to bind to the WRKY transcription factors

57 under a convincing research (Sun 2003). The proper DNA-binging ability of WRKY genes could

58 be influenced by the variation of the conserved WRKYGQK heptapeptide (Duan et al. 2007;

59 Maeo et al. 2001). 
60 The WRKY proteins can be classified into three main groups (I, II and III) on the basis of the

61 number of their WRKY domains and the pattern of the zinc finger motif. Proteins from group I

62 contain two WRKY domains followed by a $\mathrm{C}_{2} \mathrm{H}_{2}$ zinc finger motif, while the other WRKY

63 proteins from group II and III only contain one WRKY domain followed by a $\mathrm{C}_{2} \mathrm{H}_{2}$ or $\mathrm{C}_{2} \mathrm{HC}$

64 correspondingly (Yamasaki et al. 2005). Group II can be further divided into five subgroups from

65 IIa to IIe based on additional amino acid motifs present outside the WRKY domain. Apart from

66 the conserved WRKY domains and the zinc finger motif, there are some WRKY proteins

67 appearing to have basic nuclear localization signal, LZs (leucine zipper) (Cormack et al. 2002),

68 serine-threonine-rich region, glutamine-rich region and proline-rich region (Ulker \& Somssich

69 2004). Throughout the studies of WRKY gene family in many higher plants (Liu \&

70 Ekramoddoullah 2009; Rushton et al. 2010; Wu 2005), WRKY genes have been identified to be

71 involved in various regulatory processes mediated by different biotic and abiotic stresses

72 (Ramamoorthy et al. 2008). In plant defense against various biotic stresses, such as bacterial,

73 fungal and viral pathogens, it has been well documented that the WRKY genes play vital roles

74 (Cheng et al. 2015; Dong et al. 2003; He et al. 2016; Jaffar et al. 2016; Jiang et al. 2016; Kim et

75 al. 2016; Li et al. 2006; Liu et al. 2016; Xu et al. 2006; Zhou et al. 2008). They are also involved

76 in abiotic stress-induced gene expression. In Arabidopsis, with the either heat or salt treatments,

77 the expressions of AtWRKY25 and AtWRKY33 are transformed apparently (Jiang \& Deyholos

78 2009). Furthermore, the expression of TcWRKY53 that belonged to alpine penny grass (Thlaspi

79 caerulescens) is affected by salt, cold, and polyethylene glycol treatments (Wei et al. 2008). In

80 rice, a total of 54 OsWRKY genes showed noticeable differences in their transcript abundance

81 under the abiotic stress such as cold, drought, and salinity (Ramamoorthy et al. 2008). There is

82 also accumulating evidence that WRKY genes are involved in regulating developmental

83 processes, such as embryo morphogenesis (Lagace \& Matton 2004), senescence (Robatzek \&

84 Somssich 2002), trichome initiation (Johnson 2002), and some signal transduction processes 
85 mediated by plant hormones including gibberellic acid (Zhang et al. 2004), abscisic acid (Zou et

86

87

88

89

90 al. 2004), or salicylic acid (Du \& Chen 2008).

The number of WRKY genes in different species varies tremendously. For instance, there are 72 members in Arabidopsis thaliana, at least 45 in barley, 57 in cucumber, 58 in physic nut (Jatropha curcas), 59 in grapevine, 104 in poplar, 105 in foxtail millet (Setaria italica), 112 in Gossypium raimondii and more than 109 in rice (Ding et al. 2015; Eulgem 2000; Guo et al. 2014; He et al. 2012; Ling et al. 2011; Mangelsen et al. 2008; Muthamilarasan et al. 2015; Wu 2005; Xiong et al. 2013). Zhang \& Wang also identified the most basal WRKY genes in the lineage of non-plant eukaryotes and green alga (Zhang \& Wang 2005). Interestingly, the WRKY genes in eukaryotic unicellular chlamydomonas, protoctist (Giardia lambliad), bryophyte (Physcomitrella patens) and fern (Ceratopteris richardii) all belonged to group I (Di-Qiu et al. 2006; Ulker \& Somssich 2004; Zhang \& Wang 2005). For example, the study in bryophyte (Physcomitrella patens) found at least 12 WRKY genes, and all the genes belonged to group I (Ulker \& Somssich 2004). Additionally, the study in gymnosperm (Cycas revolute) identified at least 21 WRKY genes (Di-Qiu et al. 2006), and they were divided into two groups, 15 WRKY genes therein belonged to group I and the other 6 WRKY genes belonged to group II. Further study suggested that the core WRKY domains of group II and III were similar to the C-terminal domain of group I, therefore, the group II WRKY genes might emerge from the breakage of the C-terminal domain in group I and the group III probably evolve from group II (Ulker \& Somssich 2004). Above of all indicated that the group I WRKY genes might be the oldest type, which evolved from the origin of eucaryon, and group II and III might generate after the origin of bryophyte (Xie et al. 2005; Zhang \& Wang 2005). In the evolution of WRKY genes, gene duplication events played prominent roles. As a matter of fact, gene duplication events can lead to the generation of new genes. For example, there are approximately $80 \%$ of OsWRKY (rice) genes located in duplicated regions (Wu 2005), as well as $83 \%$ of PtWRKY (poplar) genes (He et al. 2012). However, no gene duplication events have occurred in cucumber (Ling et al. 2011). 
111 In the last few years, the increasing consumption of fossil fuels induced in a substantial increase

112 of $\mathrm{CO}_{2}$ concentration, which has adverse impacts on global climate changes(Pleguezuelo et al.

113 2014). Therefore, an ever-increasing demand for energy from renewable sources has provided a

114 new impetus to cultivate woody plants for bioenergy production. Due to its ease of propagation,

115 rapid growth and high yield on short rotation systems, some willow species have been used as

116 renewable resources since the 1970s. Additionally, with its essential physiological characteristics,

117 willow becomes prominent parts of the basket production, environmental restoration, analgesic

118 extraction, phytoremediation, both riparian and upland erosion control and biomass production

119 (Kuzovkina \& Quigley 2005). WRKY proteins participate in diverse physiological and

120 developmental processes in plants. With these various important factors and the recent released

121 Salix suchowensis genome sequence, which covers about 96\% of the expressed gene loci (Dai et

122 al. 2014), we have the opportunity to analyze the willow WRKY gene family. The

123 characterization of WRKY genes in willow can provide interesting gene pools to be investigated

124 for breeding and genetic engineering purposes in woody plants.

\section{Materials and Methods}

\section{Datasets and sequence retrieval}

127 The sequence of a shrub willow Salix suchowensis (S. suchowensis), which flowers within two

128 years, was conducted with a combined approach using Roche/454 and Illumina/HiSeq-2000

129 sequencing technologies (Dai et al. 2014). The latest v5.2 S. suchowensis genome annotation

130 information (version5_2.gff3) and protein sequences (Willow.gene.pep) were downloaded from

131 our laboratory website (http://bio.njfu.edu.cn/ss_wrky/). Sequences of 72 Arabidopsis WRKY

132 proteins were obtained from TAIR (release 10, http://www.arabidopsis.org/), and 104 poplar 
133 WRKY proteins were obtained from the Supplementary material 3 of poplar (Eulgem 2000; He et 134 al. 2012).

\section{Identification and distribution of WRKY genes in willow}

136 The procedure performed to identify putative WRKY proteins in willow was similar to the

137 method described in other species (Guo et al. 2014; He et al. 2012; Wu 2005). The Hidden

138 Markov Model (HMM) profile for the WRKY transcription factor was downloaded from the

139 Pfam database (http://pfam.sanger.ac.uk/) with the keyword 'PF03106' (Punta et al. 2012). The

140 HMM profile was applied as a query to search against the all willow protein sequences

141 (Willow.gene.pep) using BLASTP program (E-value cutoff =1e $\left.\mathrm{e}^{-3}\right)($ Camacho et al. 2009).

142 Another procedure was performed to validate the putative accuracy. An alignment of WRKY

143 seed sequences in Stockholm format from Pfam database was used by HMMER program

144 (hmmbuild) to build a HMM model, and then the model was used to search the willow protein

145 sequences by another HMMER program (hmmsearch) with default parameters (Eddy 1998).

146 Finally, we employed the SMART program (http://smart.embl-heidelberg.de/) to confirm the

147 candidates from the two procedures correlated with the WRKY structure features (Letunic et al. 148 2015).

149 Additionally, we calculated the length, MW (molecular weight), PI (isoelectric point) of these 150 putative WRKY proteins by ExPasy site (http://au.expasy.org/tools/pi_tool.html). Every WRKY

151 genes were mapped onto chromosomes (http://bio.njfu.edu.cn/ss_wrky/version5_2.fa) with an in-

152 house Perl script (http://bio.njfu.edu.cn/willow_chromosome/BuildGff3_Chr.pl), and then

153 renamed based on their orderly given chromosomal distribution. The distribution graph of every

154 WRKY gene was drawn by MapInspect software (http://mapinspect.software.informer.com/). 
155 Sequence alignments, phylogenetic analysis and classification of willow

156 WRKY genes

157 Using the online tool SMART, we obtained the conserved WRKY core domains of predicted

158 SsWRKY genes, and then multiple sequence alignment based on these domains was performed

159 using ClustalX (version 2.1) (Larkin et al. 2007). After alignment, we used Boxshade

160 (http://www.ch.embnet.org/software/BOX_form.html) to color the alignment result online. To

161 gain better classification of these SsWRKY genes, a further multiple sequence alignment

162 including 103 SsWRKY domains and 82 WRKY domains from Arabidopsis (AtWRKY) was

163 performed using ClustalW (Larkin et al. 2007), and a phylogenetic tree based on this alignment

164 was built by MEGA 6.0 with the Neighbor-joining (NJ) method (Tamura et al. 2013). Bootstrap

165 values have been calculated from 1000 iterations in the pairwise gap deletion mode, which is

166 conducive to the topology of the NJ tree by divergent sequences. Based on the phylogenetic tree

167 constructed by SsWRKY and AtWRKY domains, these SsWRKY genes were classified into

168 different groups and subgroups. In order to get a better comparison of WRKY family in

169 Salicaceae, a phylogenetic tree including all SsWRKY domains and 126 WRKY domains from

170 poplar (PtWRKY) was constructed with the similar method to Arabidopsis. Additionally, a

171 phylogenetic tree based on full-length SsWRKY genes was also constructed to get a better

172 classification. The ortholog of each SsWRKY gene in Arabidopsis and poplar was based on the

173 phylogenetic trees of their respective WRKY domains, and the members of group I WRKY genes

174 were considered as orthologs unless the same phylogenetic relationship can be detected between

175 N-termini and C-termini in the tree. Another method described by Zou et al., BLAST-based

176 method (Bi-direction best hit), was used to verify the putative orthologous genes (E-value cutoff

$177=1 \mathrm{e}-20)($ Chen et al. 2007; Zou et al. 2016). 


\section{Evolutionary analysis of WRKY III genes in willow}

179 The group of WRKY III genes, only found in flowering plants, is considered as the evolutionary

180 youngest groups, and plays crucial roles in the process of plant growth (He et al. 2012; Wu

181 2005). As described by Wang et al., the WRKY III genes also have a prominent impact on

182 disease and drought resistance (Wang et al. 2015). Previous study of Zhang \& Wang held the

183 opinion that duplications and diversifications were plentiful in WRKY III genes, and they

184 appeared to have confronted different selection challenges (Zhang \& Wang 2005). Phylogenetic

185 analysis of WRKY III genes was performed using MEGA6.0 with 65 WRKY III genes from

186 Arabidopsis (AtWRKY), Populus (PtWRKY), grape (VvWRKY), willow (SsWRKY) and rice

187 (OsWRKY). A NJ tree was constructed with the same method described before. Additionally, we

188 estimated the non-synonymous (Ka) and synonymous (Ks) substitution ratio of SsWRKY III

189 genes to verify whether selection pressure participated in the expansion of SsWRKY III genes.

190 Each pair of these WRKY III protein sequences was first aligned using ClustalW. The alignments

191 generated by ClustalW and the corresponding cDNA sequences were submitted to the online

192 program PAL2NAL (http://www.bork.embl.de/pal2nal/) (Suyama et al. 2006), which

193 automatically calculates Ks and Ka by the codeml program in PAML (Yang 2007).

194 Analysis of exon-intron structure, gene clusters, gene duplication events and

195 conserved motif distribution of willow WRKY genes

196 The exon-intron structures of the willow WRKY genes were obtained based on the protein

197 annotation files assembled ourselves (http://bio.njfu.edu.cn/ss_wrky/version5_2.gff3), and the

198 diagrams were obtained from the online website Gene Structure Display Server (GSDS:

199 http://gsds.cbi.pku.edu.cn/) (Hu et al. 2015). 
200 Gene clusters are very important for predicting co-expression genes or potential function of

201 clustered genes in angiosperms (Overbeek et al. 1999). They can be defined as a single

202 chromosome containing two or more genes within $200 \mathrm{~kb}$ (He et al. 2012; Holub 2001).

203 Gene duplication events were always considered as the vital sources of biological evolution.

204 Two or more adjacent homologous genes located on a single chromosome were considered as

205 tandem duplication events (TDs), while homologous gene pairs between different chromosomes

206 were defined as segmental duplication events (SDs) (Liu \& Ekramoddoullah 2009). BLASTP (E-

207 value cutoff $=1 \mathrm{e}-20$ ) was performed to identify the gene duplication events in SsWRKY genes

208 with the following definition (Gu et al. 2002; He et al. 2012): (1) the coverage of the aligned

209 sequence $\geq 80 \%$ of the longer gene; and (2) the similarity of the aligned regions $\geq 70 \%$. In this

210 study, we set the cutoff of the similarity of the aligned regions as $65 \%$, because the similarity of

211 the unaligned regions may reduce the value in different species.

212 To better exhibit the structural features of SsWRKY proteins, the online tool MEME (Multiple

213 Expectation Maximization for Motif Elicitation) was used to identify the conserved motifs in the

214 encoded SsWRKY proteins (Bailey et al. 2006). The optimized parameters were employed as the

215 following: any number of repetitions, maximum number of motifs $=20$, and the optimum width

216 of each motif was constrained to between 6 to 50 residues. The online program 2ZIP

217 (http://2zip.molgen.mpg.de/) was used to verify the existence of the conserved Leu zipper motif

218 (Bornberg-Bauer 1998), whereas some other important conserved motifs, HARF, LXXLL (X,

219 any amino acid) and LXLXLX, were identified manually.

\section{Expression analyses of willow WRKY genes}

221 The sequenced S. suchowensis RNA-HiSeq reads from five tissues including tender roots, young

222 leaves, vegetative buds, non-lignified stems and barks generated in our previous study were

223 separately mapped back onto the SsWRKY gene sequences using BWA (mismatch $\leq 2 \mathrm{bp}$, other 
224 parameters as default) (Li \& Durbin 2009), and the number of mapped reads for each WRKY

225 gene was counted. Normalization of the mapped reads was done using RPKM (reads per kilo

226 base per million reads) method (Wagner et al. 2012). The heat map for tissue-specific expression

227 profiling was generated based on the $\log _{2} \mathrm{RPKM}$ values for each gene in all the tissue samples

228 using R package (Gentleman et al. 2004).

229 Results

230 Identification and characterization of 85 WRKY genes in willow (Salix

231 suchowensis)

232 In this study, we obtained 92 putative WRKY genes by using HMMER to search the Hidden

233 Markov Model profile of WRKY DNA-binding domain against willow protein sequences, and

234 validated the accuracy of the consequence by BLASTP. After submitting the 92 putative WRKY

235 genes to the online program SMART, seven genes without a complete WRKY domain were

236 removed, while the other 85 WRKY genes were selected as possible members of the WRKY

237 superfamily.

238 WRKY genes contain one or two WRKY domains, comprising a conserved WRKYGQK

239 heptapeptide at the $\mathrm{N}$-termini and a novel zinc finger motif $\left(\mathrm{C}-\mathrm{X}_{4-7}-\mathrm{C}-\mathrm{X}_{22-23} \mathrm{H}-\mathrm{X}-\mathrm{H} / \mathrm{C}\right)$ at the $\mathrm{C}$ -

240 termini (Eulgem 2000). The variations of WRKY core domain or zinc finger motif may lead to

241 the binding specificities of WRKY genes, but this remains to be largely demonstrated (Brand et

242 al. 2013; Rinerson et al. 2015; Yamasaki et al. 2005). In order to identify the variations in WRKY

243 core domains, a multiple sequence alignment of 85 SsWRKY core domains was conducted, and

244 the result was shown in Fig. 1. Among the selected 85 WRKY genes, $81(95.3 \%)$ were identified

245 to have highly conserved sequence WRKYGQK, whereas the other four WRKY genes 
246 (SsWRKY14, SsWRKY23, SsWRKY38 and SsWRKY78) had a single mismatched amino acid

247 in their core WRKY domains (Fig. 1). In SsWRKY14 and SsWRKY38, the WRKY domain has

248 the sequence WRKYGKK, while SsWRKY23 contains a WKKYGQK sequence, and

249 SsWRKY78 contains WRKYGRK sequence. Eulgem previously described that the zinc finger

250 motif $\left(\mathrm{C}-\mathrm{X}_{4-5}-\mathrm{X}_{22-23}-\mathrm{H}-\mathrm{X}_{1}-\mathrm{H}\right.$ or $\left.\mathrm{C}-\mathrm{X}_{7}-\mathrm{C}-\mathrm{X}_{23}-\mathrm{H}-\mathrm{X}_{1}-\mathrm{C}\right)$ is another vital feature of the WRKY family

251 (Eulgem 2000). As illustrated in Fig. 1, four WRKY domains (SsWRKY76C, SsWRKY64,

252 SsWRKY12 and SsWRKY28) do not contain any distinct zinc finger motif, but they were still

253 reserved in the succeeding analyses, as performed in barley and poplar (He et al. 2012;

254 Mangelsen et al. 2008). Additionally, some zinc-finger-like motifs, including C- $\mathrm{X}_{4}-\mathrm{C}-\mathrm{X}_{21}-\mathrm{H}-\mathrm{X}_{1}$ -

$255 \mathrm{H}$ in SsWRKY23 and C-X ${ }_{5}-\mathrm{C}-\mathrm{X}_{19}-\mathrm{H}-\mathrm{X}_{1}-\mathrm{H}$ in SsWRKY73 and SsWRKY17, were identified in

256 willow WRKY genes. Both the two zinc-finger-like motifs were also found in poplar

257 (PtWRKY39, 57, 42 and 53).

258 Detailed characteristics of SsWRKY genes are listed in Table 1, including the WRKY gene

259 specific group numbers, chromosomal distribution, Arabidopsis and poplar orthologs. The

260 molecular weight (MW), isoelectric point (PI) and the length of each WRKY protein sequence

261 are also shown in Table 1. According to the particularization (Table 1), the average length of

262 these protein sequences is 407 residues, and the lengths ranged from 109 residues (SsWRKY23)

263 to 1,593 residues (SsWRKY78). Additionally, the isoelectric point (PI) ranged from 5.03

264 (SsWRKY38, SsWRKY60) to 10.27 (SsWRKY28), and the molecular weight (MW) ranged from

26512.9 (SsWRKY23) to $179.0 \mathrm{kDa}(\mathrm{SsWRKY78)}$.

266 Locations and gene clusters of willow WRKY genes

26784 of the 85 putative SsWRKY genes could be mapped onto 19 willow chromosomes and then

268 renamed from SsWRKY1 to SsWRKY84 based on their specific distributions on chromosomes.

269 Only one SsWRKY gene (willow_GLEAN_10002834), renamed as SsWRKY85, could not be 
270

271

272

273

274

275

276

277

278

279

280

281

282

283

284

285

286

287

conclusively mapped onto any chromosome. As shown in Fig. 2, Chromosome (Chr) 2 possessed the largest number of SsWRKY genes (11 genes), followed by Chr14 (10 genes). Eight SsWRKY genes were found on Chr6, six on Chr1 and Chr16, and five on Chr5. Additionally, four chromosomes (Chr4, Chr11, Chr17, Chr18) had four SsWRKY genes, as well as three SsWRKY genes were found on Chr8, Chr13 and Chr19. Chr10 and Chr15 had two SsWRKY genes, and only one SsWRKY gene was identified on Chr7, Chr9 and Chr12. The distribution of each SsWRKY genes was extremely irregular, indicating the reduction of the tandem duplication events in willow WRKY genes.

As described by Holub et al. (He et al. 2012; Holub 2001), a single chromosome region containing two or more genes within $200 \mathrm{~kb}$ was defined as gene clusters. According to this description, a total of 23 SsWRKY genes were clustered into 11 clusters in willow (Fig. 2). The chromosomal distribution of gene cluster was irregular, and only seven chromosomes were identified to have gene clusters. Three clusters, including seven SsWRKY genes, were found on Chr2, and two clusters were found on both Chr6 and Chr14. Only one cluster was distributed on each of Chr3, Chr8, Chr10 and Chr18, whereas none was identified on other eleven chromosomes. Further analysis of SsWRKY chromosomal distribution showed that a high WRKY gene density region in only 2.23 Mb regions on Chr2, which had also been observed in rice and poplar (He et al. 2012; Wu 2005).

\section{Phylogenetic analysis and classification of WRKY genes in willow}

In order to get a better separation of different groups and subgroups in SsWRKY genes, a total of 185 WRKY domains, including 82 AtWRKY domains and 103 SsWRKY domains, were used to construct the NJ phylogenetic tree. On the basis of the phylogenetic tree and structural features of WRKY domains, all 85 SsWRKY genes were clustered into three main groups (Fig. 3). Nineteen members containing two WRKY domains and $\mathrm{C}_{2} \mathrm{H}_{2}$-type zinc finger motifs were categorized into 
294 group I, except SsWRKY78, which contains only one WRKY domain and two zinc finger motifs.

295 Domain acquisition and loss events appear to have shaped the WRKY family (Ross et al. 2007;

296 Rossberg et al. 2001). Thus, SsWRKY78 may have evolved from a two-domain WRKY gene but

297 lost one WRKY domain during evolution. Additionally, as shown in Fig. 3, SsWRKY78 shows

298 high similarities to SsWRKY40N, implying a common origin of their domains. The similar

299 phenomenon was also found in PtWRKY90 of poplar (He et al. 2012).

300 The largest number of SsWRKY genes, comprising a single WRKY domain and $\mathrm{C}_{2} \mathrm{H}_{2}$ zinc

301 finger motif, were categorized into group II. SsWRKY genes of group II could be further divided 302 into five subgroups: IIa, IIb, IIc, IId and IIe. As shown in Fig. 3, subgroup IIa (4 members) and $303 \mathrm{IIb}$ (8 members) were clustered into one clade, as well as subgroup Ild (13 members) and Ie (11 304 members). Strikingly, SsWRKY genes in subgroup IIc (21 members) and group IC are classified 305 into one clade, suggesting that group II genes are not monophyletic and the group IIc WRKY 306 genes may evolve from the group I genes by the loss of the WRKY domain in N-terminal. As shown in Fig. 3 and Fig. S1, SsWRKY23, SsWRKY34 and their orthologous genes (AtWRKY49, PtWRKY39, PtWRKY57, PtWRKY34 and PtWRKY32) seem to form a new subgroup closer to the group III. However, SsWRKY23 and SsWRKY34 exhibit the zinc finger motif $\mathrm{C}-\mathrm{X}_{4}-\mathrm{C}-\mathrm{X}_{21}-\mathrm{H}-\mathrm{X}-\mathrm{H}$ and $\mathrm{C}-\mathrm{X}_{4}-\mathrm{C}-\mathrm{X}_{23}-\mathrm{H}-\mathrm{X}-\mathrm{H}$ as observed in the subgroup IIc and group IC.

311 Thereby, they were classified into subgroup IIc in this study.

312 Different from the $\mathrm{C}_{2} \mathrm{H}_{2}$ zinc finger pattern in group I and II, group III WRKY genes (7

313 members), broadly considered as playing vital roles in plant evolution process and adaptability, 314 contained one WRKY domain and a C-X $-\mathrm{C}-\mathrm{X}_{23}-\mathrm{H}-\mathrm{X}-\mathrm{C}$ zinc finger motif. However, in rice and 315 barley, a new $\mathrm{CX}_{7} \mathrm{CX}_{\mathrm{n}} \mathrm{HX}_{1} \mathrm{C}(\mathrm{n} \geq 24)$ zinc finger motif was identified in group III (Mangelsen et 316 al. 2008; Wu 2005), which was never found in poplar, grape, Arabidopsis and willow, suggesting 317 that this feature perhaps only belong to monocotyledonous species.

318 In order to obtain a better study in woody plant species, a phylogenetic tree based on the WRKY 319 domains between willow and poplar was constructed (Fig. S1). The tree showed that most of the 
320 WRKY domains from willow and poplar were clustered into sister pairs, suggesting that gene

321 duplication events played prominent roles in the evolution and expansion of WRKY gene family.

322 Furthermore, a total of twenty SsWRKY domains show extremely the same domains (similarity:

$323100 \%$ ) to poplar, i.e., SsWRKY39 and PtWRKY9, SsWRKY39 and PtWRKY9, SsWRKY39 and

324 PtWRKY9, SsWRKY39 and PtWRKY9, and so on. Further functional analyses of these genes in

325 willow or poplar will provide a useful reference for another one.

\section{The ortholog of SsWRKY genes in Arabidopsis and poplar}

327 The clustering of orthologous genes emphasizes the conservation and divergence of gene

328 families, and they may contain the same functions (Ling et al. 2011). In this study, a phylogeny-

329 based method was used to identify the putative orthologous SsWRKY genes in Arabidopsis and

330 poplar (Fig. 3 and Fig. S1), and BLAST-based method (Bi-direction best hit) was used to confirm

331 the true orthologs. The WRKY genes of group I contained two WRKY domains, and both of

332 them were used to construct the phylogenetic trees. To avoid the mistakes of orthologous genes

333 in group I, the members of group I WRKY genes were considered as orthologous genes unless

334 the same phylogenetic relationship can be detected between $\mathrm{N}$-termini and C-termini in the

335 phylogenetic tree. For example, SsWRKY37 and AtWRKY44 were considered as an orthologous

336 gene pair because they clustered into a clade of their N-termini and C-termini (Fig. 3), while

337 SsWRKY80 and PtWRKY30 were excluded from orthologous gene pairs due to their different

338 clusters of N-termini and C-termini (Fig. S1). Totally, 75 orthologous gene pairs were found

339 between willow and Arabidopsis, less than 82 orthologous gene pairs between willow and poplar

340 (Table 1), which was congruent with the evolutionary relationship among the three plant species. 


\section{Evolutionary analysis of WRKY III genes in willow}

342 The WRKY III genes were considered as the evolutionary youngest groups, and played crucial

343 roles in the process of plant growth and resistance. In order to further probe the duplication and

344 diversification of WRKY III genes after the divergence of the monocots and dicots, a

345 phylogenetic tree was constructed using 65 WRKY III genes from Arabidopsis (13), rice (29),

346 poplar (10), willow (7) and grape (6). As shown in Fig. S2, willow SsWRKY III genes were

347 closer to the eurosids I group (poplar and grape) than eurosids II group (Arabidopsis) and

348 monocots (rice). Meanwhile, most Arabidopsis and rice WRKY III genes formed the relatively

349 independent clades, suggesting that two gene duplication events, including tandem and segmental

350 duplication, perhaps were the main factors in the expansion of WRKY III genes in Arabidopsis

351 and rice. The results also indicated that WRKY III genes might arise after the divergence of the

352 Arabidopsis (eurosids I) and eurosids II (poplar, willow and grape). The study by Ling et al. in

353 cucumber (Ling et al. 2011) showed the similar results and hence proved the validity.

354 Additionally, we found that seven rice WRKY III genes (OsWRKY55, 84, 18, 52, 46, 114 and

355 97) contained the variant domain WRKYGEK, which was not found in other four dicots

356 (Arabidopsis, poplar, grape and willow), implying that this may be a feature of WRKY III genes

357 in monocots and these OsWRKY genes may respond to different environmental signals.

358 According to the comparison of the number of WRKY III genes in the five observed plants, the

359 number is smaller in eurosids I (poplar, grape and willow) than Arabidopsis (eurosids II) and rice

360 (monocots), which may be caused by different patterns of duplication events. Genes generated by

361 duplication events are not stable, and can be retained or lost due to different selection pressure

362 and evolution (Zhang 2003). In order to determine which selection pressure played prominent

363 roles in the expansion of willow WRKY III genes, we estimated the Ka/Ks ratios for all pairs (21

364 pairs) of willow WRKY III genes. As shown in Table S1, all the Ka/Ks ratios were less than 0.5, 
365 suggesting willow WRKY III genes had mainly been subjected to strong purifying selection and 366 they were slowing evolving at the protein level.

\section{Exon-intron structures of SsWRKY genes}

368 The exon-intron structures of multiple gene families play crucial roles during plant evolution. As

369 shown in Fig. 4, the SsWRKY gene phylogenetic tree and the corresponding exon-intron

370 structures are shown in A and B, respectively. Exon-intron structures of each group were shown

371 in Fig. 4B, a large number of WRKY genes had two to five introns (94\%, 80 of 85), including 8

372 WRKY genes contained one intron; 39 contained two introns; 13 contained three introns; 15

373 contained four introns and 5 contained five introns. The number of exons in remaining WRKY

374 genes was quite different: SsWRKY49, SsWRKY76 and SsWRKY78 had six, eleven and ten

375 introns, respectively; SsWRKY17 had the largest number of introns (seventeen introns), while no

376 intron was found in SsWRKY12. The intron acquisition or loss occurred during the evolution of

377 WRKY gene family, while WRKY genes in the same group shared the similar number of introns

378 (Guo et al. 2014). In our study, most of WRKY genes in group I had three to six introns, expect

379 SsWRKY76 and SsWRKY78, which might acquire some introns during evolution. The number

380 of introns of WRKY genes in group II was extremely different, ranging from one to five introns,

381 except SsWRKY17 with 17 introns and SsWRKY12 with zero intron might obtain or loss some

382 introns during evolution. Strikingly, WRKY genes in group III had the most stable number of

383 introns with all of seven WRKY III genes had two introns, suggesting that WRKY III genes may

384 be the most stable genes in the environmental stress. The stable number of introns in SsWRKY

385 III genes was consistent with the results of $\mathrm{Ka} / \mathrm{Ks}$ analysis, which reflected that purifying

386 selection pressure played vital roles in willow WRKY III genes.

387 A great deal of studies in WRKY genes proved that nearly all of the WRKY genes contained an

388 intron in their WRKY core domains (Eulgem 2000; Guo et al. 2014; He et al. 2012; Huang et al. 
389

390

391

392

393

394

395

396

397

398

399

400

401

402

403

404

405

406

407

408

409

410

411

412

2012; Ling et al. 2011; Zou et al. 2004). According to the further analysis of SsWRKY genes, two major types of splicing introns, R-type and V-type, introns were observed in numerous

SsWRKY domains. The R-type intron was spliced exactly at the $\mathrm{R}$ residue, about five amino acids before the first Cys residue in the $\mathrm{C}_{2} \mathrm{H}_{2}$ zinc finger motif. The $\mathrm{V}$-type intron was localized before the $V$ residue, six amino acids after the second Cys residue in the $\mathrm{C}_{2} \mathrm{H}_{2}$ zinc finger motif. As shown in Fig. 4B, the R-type introns could be observed in more groups, including group IC, subgroup IIc, IId, IIe and group III, while V-type introns were only observed in subgroup IIa and IIb. However, there was no intron found in group IN. The similar results were also observed in Arabidopsis, poplar and rice, suggesting that the special distribution of introns in WRKY domains was a feature of WRKY family (Eulgem 2000; He et al. 2012; Wu 2005).

\section{Identification of gene duplication events and conserved motifs in willow}

Gene duplication events were always considered as the vital sources of biological evolution (Chothia et al. 2003; Ohno et al. 2009). TDs were defined as two or more adjacent homologous genes located on a single chromosome, while homologous gene pairs between different chromosomes were defined as SDs (Liu \& Ekramoddoullah 2009). In our study, a total of 33 homologous gene pairs, including 66 SsWRKY genes, were identified to participate in gene duplication events (Table S2). The composition of gene duplication events in each group in ascending order was group I: 73.7\% (14 of 19), group II: $78 \%$ (46 of 59) and group III: 85.7\% (6 of 7). Among the 33 homologous gene pairs, none of them appeared to have undergone TDs, on the contrary, all of the 66 genes (77.6\% of all SsWRKY genes) participated in SDs, implying that segmental duplication events played major roles in the expansion of willow WRKY genes.

WRKY genes shared more functional and homologies in their conserved WRKY core domains (about 60 residues), while the rest sequences of WRKY genes shared a little (Eulgem 2000). In order to get a more comprehensive understanding of the structural feature in WRKY domains, the 
413 conserved motifs of SsWRKY genes were predicted using the online program MEME (Fig. S3

414 and Table S3). Among the 20 putative motifs, motifs 1, 2, 3 and 5, broadly distributed across

415 SsWRKY genes, were characterized as the WRKY conserved domains. The motif 6 was

416 characterized as nuclear localization signals (NLS), which mainly distributed in subgroup II d

417 and IIe and group III. Some other motifs with poorly defined recently were also predicted by

418 MEME: the motif 4 was only found in group IC and subgroup IIc; motifs 7 and 9 were limited to

419 subgroup IIa and IIb; the motif 8 was found in group I and a few genes of subgroup IIc; motifs

$42010,13,15$ and 17 were unique in subgroup IId; the motif 12 was only observed in subgroup IIb;

421 the motif 16 was mainly found in group II; the motif 18 was found in subgroup IIc; motifs 19 and

42220 were only observed in subgroup I. The distinct conserved motifs of different groups could be

423 an important foundation for future structural and functional study in WRKY gene family.

424 Some other important motifs, including Leu zipper motif, HARF, LXXLL and LXLXLX, could

425 be also identified in WRKY genes. Using the online program 2ZIP, the conserved Leu zipper

426 motif, described as a common hypothetical structure to DNA binding proteins (McInerney et al.

427 1998), was identified in only two SsWRKY genes (SsWRKY61 and SsWRKY39). With manual

428 inspection, the conserved HARF (RTGHARFRR[A/G]P) motifs, whose putative functions were

429 not distinguished clearly, were only observed in seven WRKY genes of subgroup IId, including

430 SsWRKY82, 33, 45, 81, 9, 30 and 56. In the meantime, the conserved LXXLL and LXLXLX (L:

431 Leucine; X: any amino acid) motifs, which respectively defined as the co-activator and active

432 repressor motifs, were also found in SsWRKY genes. A total of seven SsWRKY genes

433 (SsWRKY19, 45, 72, 61, 76, 30 and 59) contained the helical motif LXXLL, whereas eight genes

434 (SsWRKY66, 26, 35, 81, 83, 75, 73 and 3) shared the LXLXLX motif. The plenty of conserved

435 motifs in WRKY genes with different lengths and variant functions, suggesting that the WRKY

436 genes might play more vital roles in gene regulatory network. 


\section{Distinct expression profiles of SsWRKY genes in various tissues}

438 In order to gain more information about the roles of WRKY genes in willow, RNA-seq data from

439 the sequenced genotype were used to quantify the expression level of WRKY genes in five

440 tissues of Salix suchowensis. As illustrated in Fig. 5, the expression of all 85 SsWRKY genes

441 were detected in at least one of the five examined tissues, such as 84 genes in roots, 80 in stems,

44284 in barks, all in buds and 73 in leaves. Meanwhile, the cluster analysis of the expression pattern

443 in five tissues showed that SsWRKY genes shared more similarities between stem and leaf, as

444 well as bark and bud, and root was more similar to the clade formed by bark and bud. The results

445 detected here were consistent with their biological characteristics. SsWRKY38, not detected in

446 roots and leaves, was also lowly expressed in other tissues. Similarly, SsWRKY74, not detected

447 in stems, barks and leaves, was only expressed in roots and buds with extremely low levels.

448 Among the five genes not expressed in stems, SsWRKY66, 74 and 79 were also not detected in

449 leaves. The largest number of expressed or unexpressed SsWRKY genes (12 genes) was found in

450 buds or leaves, respectively, suggesting that WRKY genes might play more roles in buds than

451 leaves.

452 According to the expression annotation of 85 SsWRKY genes by RPKM method in Fig. 5 and

453 Table S4, the total transcript abundance of SsWRKY genes in tender root (RPKM = 1181.21),

454 bark $(\mathrm{RPKM}=1363.01)$ and vegetative bud $(\mathrm{RPKM}=928.58)$ was relatively larger than that in

455 other two tissues, including non-lignified stem $(\mathrm{RPKM}=537.88)$ and young leaf $(\mathrm{RPKM}=$

456 349.84). As shown in Table S4, SsWRKY81 (RPKM = 97.75), the most expressed SsWRKY

457 genes in roots, was also expressed in other four tissues, though the expression levels were

458 relatively low; SsWRKY56 $($ RPKM = 32.54), the most expressed SsWRKY genes in stem, was

459 also highly expressed in other examined tissues. Similarly, SsWRKY67, the most expressed

460 SsWRKY genes in barks (RPKM = 188.16), was also detected in vegetative buds (RPKM

$461=82.07)$ and young leaves $(\mathrm{RPKM}=26.11)$ with high expression levels. Similarly, SsWRKY6 
$462(\mathrm{RPKM}=26.31)$, the most expressed genes in leaves, was also highly detected in other tissues. A

463 few genes, i.e., SsWRKY52, SsWRKY2 and SsWRKY35, were expressed highly in barks, but

464 lowly in other four tissues. The results mentioned above may be an important foundation for the

465 specific expression analysis of each WRKY gene in willow.

\section{Discussion}

467 The WRKY transcription factor gene family can specifically interact with the W-box to regulate

468 the expressions of downstream target genes. They also play prominent roles in diverse

469 physiological and growing processes, especially in various abiotic and biotic stress responses in

470 plants. Previous studies about the features and functions of WRKY family have been conducted

471 in many model plants, including Arabidopsis for annual herbaceous dicots (Eulgem 2000), grape

472 for perennial dicots (Guo et al. 2014), poplar for woody plants and rice for monocots (He et al.

473 2012; Wu 2005), but there is no large-scale study of WRKY genes in willow. Here, the

474 comprehensive analysis of WRKY family in willow (Salix suchowensis) would facilitate a better

475 understanding of WRKY gene superfamily and provide interesting gene pools to be investigated

476 for breeding and genetic engineering purposes in woody plants.

477 As described in many previous studies, the presence of highly conserved WRKY domains in

478 WRKY proteins is the most prominent characteristic of the WRKY gene family (Ding et al.

479 2015; Eulgem 2000; He et al. 2012; Huang et al. 2012; Wu 2005). In our study, through

480 comparing the two phylogenetic trees based on the conserved WRKY domains (Fig. 3) and

481 proteins (Fig. 4A), we obtained the nearly same classification of all SsWRKY genes, suggesting

482 that the conserved WRKY domain is an indispensable unit in WRKY genes. The variation of the

483 WRKYGQK heptapeptide may influence the proper DNA-binging ability of WRKY genes (Duan

484 et al. 2007; Maeo et al. 2001). A recent binding study by Brand et al. disclosed that a reciprocal

$485 \mathrm{Q} / \mathrm{K}$ change of the WRKYGQK heptapeptide might result in different DNA-binding specificities 
486 of the respective WRKY genes (Brand et al. 2013). For instance, the soybean WRKY genes,

487 GmWRKY6 and GmWRKY21, which contains the WRKYGKK variant, can't bind normally to

488 the W-box(Zhou et al. 2008). NtWRKY12 gene in tobacco with the WRKYGKK variant

489 recognizes another binding sequence 'TTTTCCAC' instead of normal W-box (van Verk et al.

490 2008). In our study, four WRKY genes (SsWRKY14, SsWRKY23, SsWRKY38 and

491 SsWRKY78) had a single mismatched amino acid in their conserved WRKYGQK heptapeptide

492 (Fig. 1). The variants detected in willow were extremely congruent with that in another

493 salicaceous plant, poplar, which also contains the same three variants in seven PtWRKY genes

494 (He et al. 2012). Previous studies have disclosed that the binding specificities of variable

495 WRKYGQK heptapeptide vary tremendously (Brand et al. 2013), however, few studies were

496 shown about the effect of variable zinc finger motif. In this study, four WRKY domains

497 (SsWRKY76C, SsWRKY64, SsWRKY12 and SsWRKY28) without complete zinc finger motif

498 may lack the ability of interacting with W-box, as well as PtWRKY83, 40, 95 and 10 in poplar

499 (He et al. 2012). Thereby, it is still indispensable to further investigate the function or the

500 expression patterns of the regulated gene targets in the variant sequences of the WRKY domains

501 (both WRKYGQK heptapeptide and complete zinc finger motif).

502 Different classification methods may lead to different numbers of WRKY genes in each group.

503 The classification method in our study was categorized as described in Arabidopsis, grape,

504 cucumber, castor bean and many other plant species (Eulgem 2000; Guo et al. 2014; Ling et al.

505 2011; Zou et al. 2016). According to this method, the willow WRKY genes were classified into

506 three main groups (I, II and III), with five subgroups in group II (IIa, IIb, IIc, IId and IIe).

507 However, the strategy described in rice and poplar was a little different (He et al. 2012; $\mathrm{Wu}$

508 2005). They classified the subgroup IIc categorized above into a new subgroup Ib based on the

509 fact that the C-termini of group I and the domains of the above subgroup IIc shared more similar

510 consensus structures. At the meantime, subgroup IId and IIe categorized above were reclassified

511 into subgroup IIc and IId, respectively. With the same classification method as described in 
512 Arabidopsis and many other plants, the numbers of different groups in poplar and rice are

513 illustrated in Table S5. WRKY genes of subgroup IIa, the smallest number of members, appear to

514 play crucial roles in regulating biotic and abiotic stress responses (Rushton et al. 2010). As shown

515 in Table S5, the willow WRKY genes of subgroup IIa and IIb are extremely similar to that of

516 other plant species, suggesting that all SsWRKY genes of these subgroups have been identified.

517 In addition, the numbers of WRKY III in eurosids I group, such as cucumber (6), poplar (10),

518 grape (6) and willow (7) are less than that of eurosids II (Arabidopsis: 14) and monocots (rice:

519 36), suggesting that different duplication events or selection pressures occurred in WRKY III

520 genes after the divergence of eurosids I and eurosids II group. A previous study in Arabidopsis

521 showed that nearly all WRKY III members respond to diverse biotic stresses, indicating that this

522 group probably evolved with the increasing biological requirements (Wang et al. 2015). The

523 different numbers of WRKY III genes in willow, poplar, cucumber, Arabidopsis and rice are

524 probably due to their different biotic stresses during evolution, and seven SsWRKY III genes

525 may be sufficient for the biological requirements in willow.

526 WRKY transcription factors play important roles in the regulation of developmental processes

527 and response to biotic and abiotic stress (Brand et al. 2013). The evolutionary relationship of

528 WRKY gene family promises to obtain significant insights into how biotic and abiotic stress

529 responses from single cellular aquatic algae to multicellular flowering plants (Rinerson et al.

530 2015). Previous studies hypothesized that group I WRKY genes were generated by domain

531 duplication of a proto-WRKY gene with a single WRKY domain, group II WRKY genes evolved

532 through the subsequent loss of N-terminal WRKY domain, and group III genes evolved from the

533 replacement of conserved His residue with a Cys residue in zinc motif (Wu 2005). However,

534 recent study proposed two alternative hypotheses of WRKY gene evolution (Rinerson et al.

535 2015): the "Group I Hypothesis" and the "IIa + b Separate Hypothesis". Additionally, another

536 recent study by Brand et al. concluded that subgroup IIc WRKY genes evolved directly from IIc-

537 like ancestral WRKY domains, and group I genes evolved independently due to a duplication of 
538 the IIc-like ancestral WRKY domains (Brand et al. 2013). Phylogenetic analysis in our study

539 shows that subgroup IIc and group IC are evolutionarily close, as well as subgroups IIa and IIb,

540 subgroups IId and IIe, and this result is consistent with the conclusion drew by Brand et al.

541 (Brand et al. 2013). Additionally, the V-type introns of SsWRKY genes are only found in

542 subgroup IIa and IIb, while R-type introns are found in other groups except group IN. The results

543 are congruent with the "IIa + b Separate Hypothesis". Our results shown here provide important

544 reference for the further analyses on the accurate evolutionary relationship of WRKY gene

545 family.

546 Gene duplication events played prominent roles in a succession of genomic rearrangements and 547 expansions, and it is also the main motivation of plants evolution (Vision et al. 2000). The gene

548 family expansion occurs via three mechanisms: TDs, SDs and transposition events (Maher et al.

549 2006), and we only focused on the TDs and SDs in this study. In willow, a total of 66 SsWRKY

550 genes were identified to participate in gene duplication events, and all of these genes appeared to

551 have undergone SDs. Similarly, in poplar, only one homologous gene pair participated in TDs,

552 while 29 of $42(69 \%)$ homologous gene pairs were determined to participate in SDs. The similar

553 WRKY gene expansion patterns in willow and poplar showed that SDs were the main factors in

554 the expansion of WRKY genes in woody plants. However, in cucumber, no gene duplication

555 events have occurred in CsWRKY gene evolution, probably because there were no recent whole-

556 genome duplication and tandem duplication in cucumber genome (Huang et al. 2009). In rice and

557 Arabidopsis, many WRKY genes were generated by TDs, which was incongruent with the

558 duplication events in willow, poplar and cucumber. The different WRKY gene expansion

559 patterns of the above plant species could be due to their different life habits and selection

560 pressures in a large scale, and it is still indispensable to be further investigated.

561 The WRKY gene family plays crucial roles in response to biotic and abiotic stresses, as well as

562 diverse physiological and developmental processes in plant species. Because of the lack of

563 researches on the function of willow WRKY genes, our study provided putative functions of 
564 SsWRKY genes by comparing the orthologous genes between willow and Arabidopsis. The

565 details of the functions or regulations of AtWRKY genes can be obtained from TAIR

566 (http://www.arabidopsis.org/). For example, AtWRKY2, the ortholog to SsWRKY6, which

567 highly expressed in the five examined tissues, plays important roles in seed germination and post

568 germination growth (Jiang \& Yu 2009). AtWRKY33, the ortholog to SsWRKY1, 35, 55 and 84,

569 influences the tolerance to $\mathrm{NaCl}$, and increases sensitivity to oxidative stress and abscisic acid

570 (Jiang \& Deyholos 2009). A large number of AtWRKY genes, i.e. AtWRKY3, 4, 18, 53, 41,

571 work in the resistance to Pseudomonas syringae (Chen \& Chen 2002; Higashi et al. 2008; Lai et

572 al. 2008; Murray et al. 2007), therefore, their orthologs in willow (SsWRKY42, 47, 39, 79, 20

573 and 70) may show the same resistance to Pseudomonas syringae. Based on the comparison of

574 willow WRKY genes with their Arabidopsis orthologs, we could speculate that the functional

575 divergence of SsWRKY genes has played prominent roles in the responses to various stresses.

\section{Conclusions}

577 Based on the recent released willow genome sequence and RNA-seq data, in this study, we

578 identified 85 SsWRKY proteins using bioinformatics approach. According to the phylogenetic

579 relationships and structural features of WRKY domains, all 85 SsWRKY genes were assigned to

580 the group I, group II (subgroup a-e) and group III. Three variations of the WRKYGQK

581 heptapeptide and the normal zinc finger motif in willow WRKY genes might execute some new

582 biological functions. Evolutionary analysis of SsWRKY III genes will be helpful for

583 understanding the evolution of WRKY III genes in plant. With the comparison of willow WRKY

584 genes with their Arabidopsis orthologs, breeding willow varieties with increased tolerance to

585 many adverse environments could be achieved using transgenic technology. Our results will be

586 not only beneficial to complete the functional and annotation information of WRKY genes family 
587 in woody plants, but also provide interesting gene pools to be investigated for breeding and

588 genetic engineering purposes in woody plants.

\section{Acknowledgments}

590 The authors are deeply grateful to Prof. Tongming Yin, who provided the draft willow genome

591 and RNA-seq data from five tissues. The authors also thank Prof. Ning Ye, Prof. Qiaolin Ye and

592 Dr. Yiqing Xu for providing valuable suggestions and comments.

\section{References}

594

595

596

597

598

599

600

601

602

603

604

605

606

607

608

609

610

611

612

613

614

615
Bailey TL, Williams N, Misleh C, and Li WW. 2006. MEME: discovering and analyzing DNA and protein sequence motifs. Nucleic Acids Res 34:W369-373. 10.1093/nar/gkl198

Bornberg-Bauer E. 1998. Computational approaches to identify leucine zippers. Nucleic Acids Res 26:2740-2746. 10.1093/nar/26.11.2740

Brand LH, Fischer NM, Harter K, Kohlbacher O, and Wanke D. 2013. Elucidating the evolutionary conserved DNA-binding specificities of WRKY transcription factors by molecular dynamics and in vitro binding assays. Nucleic Acids Res 41:9764-9778. 10.1093/nar/gkt732

Camacho C, Coulouris G, Avagyan V, Ma N, Papadopoulos J, Bealer K, and Madden TL. 2009. BLAST+: architecture and applications. BMC Bioinformatics 10:421. 10.1186/14712105-10-421

Chen C, and Chen Z. 2002. Potentiation of developmentally regulated plant defense response by AtWRKY18, a pathogen-induced Arabidopsis transcription factor. Plant Physiol 129:706716. 10.1104/pp.001057

Chen F, Mackey AJ, Vermunt JK, and Roos DS. 2007. Assessing performance of orthology detection strategies applied to eukaryotic genomes. PLoS One 2:e383. 10.1371/journal.pone.0000383

Cheng H, Li H, Deng Y, Xiao J, Li X, and Wang S. 2015. The WRKY45-2-WRKY13-WRKY42 Transcriptional Regulatory Cascade Is Required for Rice Resistance to Fungal Pathogen. Plant Physiol 167:1087-1099.

Chothia C, Gough J, Vogel C, and Teichmann SA. 2003. Evolution of the protein repertoire. Science 300:1701-1703. 10.1126/science.1085371 
616 Ciolkowski I, Wanke D, Birkenbihl RP, and Somssich IE. 2008. Studies on DNA-binding 617 selectivity of WRKY transcription factors lend structural clues into WRKY-domain 618 function. Plant Mol Biol 68:81-92. 10.1007/s11103-008-9353-1

619 Cormack RS, Eulgem T, Rushton PJ, Köchner P, Hahlbrock K, and Somssich IE. 2002. Leucine 620 zipper-containing WRKY proteins widen the spectrum of immediate early elicitor621 induced WRKY transcription factors in parsley. Biochimica et Biophysica Acta (BBA) $622 \quad$ Gene Structure and Expression 1576:92-100. 10.1016/s0167-4781(02)00298-1

623 Dai X, Hu Q, Cai Q, Feng K, Ye N, Tuskan GA, Milne R, Chen Y, Wan Z, Wang Z, Luo W, Wang 624 K, Wan D, Wang M, Wang J, Liu J, and Yin T. 2014. The willow genome and divergent 625 evolution from poplar after the common genome duplication. Cell Res 24:1274-1277. $626 \quad 10.1038 / \mathrm{cr} .2014 .83$

627 Di-Qiu YU, Chen LG, and Zhang LP. 2006. Transcription Factor WRKY Superfamily:Origin, 628 Structure and Function. Acta Botanica Yunnanica 28:69-77.

Ding M, Chen J, Jiang Y, Lin L, Cao Y, Wang M, Zhang Y, Rong J, and Ye W. 2015. Genomewide investigation and transcriptome analysis of the WRKY gene family in Gossypium. Mol Genet Genomics 290:151-171. 10.1007/s00438-014-0904-7

Dong J, Chen C, and Chen Z. 2003. Expression profiles of the ArabidopsisWRKY gene superfamily during plant defense response. Plant Mol Biol 51:21-37. 10.1023/a:1020780022549

Du L, and Chen Z. 2008. Identification of genes encoding receptor-like protein kinases as possible targets of pathogen- and salicylic acid-induced WRKY DNA-binding proteins in Arabidopsis. The Plant Journal 24:837-847. 10.1111/j.1365-313X.2000.00923.X

Duan MR, Nan J, Liang YH, Mao P, Lu L, Li L, Wei C, Lai L, Li Y, and Su XD. 2007. DNA binding mechanism revealed by high resolution crystal structure of Arabidopsis thaliana WRKY1 protein. Nucleic Acids Res 35:1145-1154. 10.1093/nar/gkm001

Eddy SR. 1998. Profile hidden Markov models. Bioinformatics 14:755-763. 10.1093/bioinformatics/14.9.755

Eulgem T. 2000. The WRKY superfamily of plant transcription factors. Trends in Plant Science 5:199-206. 10.1016/s1360-1385(00)01600-9

Gentleman RC, Carey VJ, Bates DM, Bolstad B, Dettling M, Dudoit S, Ellis B, Gautier L, Ge Y, Gentry J, Hornik K, Hothorn T, Huber W, Iacus S, Irizarry R, Leisch F, Li C, Maechler M, Rossini AJ, Sawitzki G, Smith C, Smyth G, Tierney L, Yang JY, and Zhang J. 2004.

652

653 Bioconductor: open software development for computational biology and bioinformatics. Genome Biol 5:R80. 10.1186/gb-2004-5-10-r80

Giacomelli JI, Ribichich KF, Dezar CA, and Chan RL. 2010. Expression analyses indicate the involvement of sunflower WRKY transcription factors in stress responses, and phylogenetic reconstructions reveal the existence of a novel clade in the Asteraceae. Plant Science 178:398-410. 10.1016/j.plantsci.2010.02.008 
654 Gu ZL, Cavalcanti A, Chen FC, Bouman P, and Li WH. 2002. Extent of gene duplication in the 655 genomes of Drosophila, nematode, and yeast. Molecular Biology and Evolution 19:256656262.

657 Guo C, Guo R, Xu X, Gao M, Li X, Song J, Zheng Y, and Wang X. 2014. Evolution and 658 expression analysis of the grape (Vitis vinifera L.) WRKY gene family. $J$ Exp Bot 659 65:1513-1528. 10.1093/jxb/eru007

660 He GH, Xu JY, Wang YX, Liu JM, Li PS, Chen M, Ma YZ, and Xu ZS. 2016. Drought661 responsive WRKY transcription factor genes TaWRKY1 and TaWRKY33 from wheat confer drought and/or heat resistance in Arabidopsis. BMC Plant Biol 16:1-16.

He H, Dong Q, Shao Y, Jiang H, Zhu S, Cheng B, and Xiang Y. 2012. Genome-wide survey and characterization of the WRKY gene family in Populus trichocarpa. Plant Cell Rep 31:1199-1217. 10.1007/s00299-012-1241-0

Higashi K, Ishiga Y, Inagaki Y, Toyoda K, Shiraishi T, and Ichinose Y. 2008. Modulation of defense signal transduction by flagellin-induced WRKY41 transcription factor in Arabidopsis thaliana. Mol Genet Genomics 279:303-312. 10.1007/s00438-007-0315-0

Holub EB. 2001. The arms race is ancient history in Arabidopsis, the wildflower. Nat Rev Genet 2:516-527. 10.1038/35080508

Hu B, Jin J, Guo AY, Zhang H, Luo J, and Gao G. 2015. GSDS 2.0: an upgraded gene feature visualization server. Bioinformatics 31:1296-1297. 10.1093/bioinformatics/btu817

Huang S, Gao Y, Liu J, Peng X, Niu X, Fei Z, Cao S, and Liu Y. 2012. Genome-wide analysis of WRKY transcription factors in Solanum lycopersicum. Mol Genet Genomics 287:495513. 10.1007/s00438-012-0696-6

Huang S, Li R, Zhang Z, Li L, Gu X, Fan W, Lucas WJ, Wang X, Xie B, Ni P, Ren Y, Zhu H, Li J, Lin K, Jin W, Fei Z, Li G, Staub J, Kilian A, van der Vossen EA, Wu Y, Guo J, He J, Jia Z, Ren Y, Tian G, Lu Y, Ruan J, Qian W, Wang M, Huang Q, Li B, Xuan Z, Cao J, Asan, Wu Z, Zhang J, Cai Q, Bai Y, Zhao B, Han Y, Li Y, Li X, Wang S, Shi Q, Liu S, Cho WK, Kim JY, Xu Y, Heller-Uszynska K, Miao H, Cheng Z, Zhang S, Wu J, Yang Y, Kang H, Li M, Liang H, Ren X, Shi Z, Wen M, Jian M, Yang H, Zhang G, Yang Z, Chen R, Liu S, Li J, Ma L, Liu H, Zhou Y, Zhao J, Fang X, Li G, Fang L, Li Y, Liu D, Zheng H, Zhang Y, Qin N, Li Z, Yang G, Yang S, Bolund L, Kristiansen K, Zheng H, Li S, Zhang X, Yang H, Wang J, Sun R, Zhang B, Jiang S, Wang J, Du Y, and Li S. 2009. The genome of the cucumber, Cucumis sativus L. Nat Genet 41:1275-1281. 10.1038/ng.475

Ishiguro S, and Nakamura K. 1994. Characterization of a cDNA encoding a novel DNA-binding protein, SPF1, that recognizes SP8 sequences in the 5' upstream regions of genes coding for sporamin and ß-amylase from sweet potato. MGG Molecular \& General Genetics 244. 10.1007/bf00282746

Jaffar MA, Song A, Faheem M, Chen S, Jiang J, Liu C, Fan Q, and Chen F. 2016. Involvement of CmWRKY10 in Drought Tolerance of Chrysanthemum through the ABA-Signaling Pathway. International Journal of Molecular Sciences 17. 
693 Jang J-Y, Choi C-H, and Hwang D-J. 2010. The WRKY Superfamily of Rice Transcription

694

695

696

697

698

699

700

701

702

703

704

705

706

707

708

709

710

711

712

713

714

715

716

717

718

719

720

721

722

723

724

725

726

727

728

729

730 Factors. The Plant Pathology Journal 26:110-114. 10.5423/ppj.2010.26.2.110

Jiang M, Liu QE, Liu ZN, Li JZ, and He CM. 2016. Over-expression of a WRKY transcription factor gene BoWRKY6 enhances resistance to downy mildew in transgenic broccoli plants. Australasian Plant Pathology:1-8.

Jiang W, and Yu D. 2009. Arabidopsis WRKY2 transcription factor mediates seed germination and postgermination arrest of development by abscisic acid. BMC Plant Biol 9:96. 10.1186/1471-2229-9-96

Jiang Y, and Deyholos MK. 2009. Functional characterization of Arabidopsis NaCl-inducible WRKY25 and WRKY33 transcription factors in abiotic stresses. Plant Mol Biol 69:91105. 10.1007/s11103-008-9408-3

Johnson CS. 2002. TRANSPARENT TESTA GLABRA2, a Trichome and Seed Coat Development Gene of Arabidopsis, Encodes a WRKY Transcription Factor. The Plant Cell Online 14:1359-1375. 10.1105/tpc.001404

Kim CY, Vo KTX, Cong DN, Jeong DH, Lee SK, Kumar M, Kim SR, Park SH, Kim JK, and Jeon JS. 2016. Functional analysis of a cold-responsive rice WRKY gene, OsWRKY71. Plant Biotechnology Reports 10:13-23.

Kuzovkina YA, and Quigley MF. 2005. Willows Beyond Wetlands: Uses of Salix L. Species for Environmental Projects. Water Air \& Soil Pollution 162:183-204.

Lagace M, and Matton DP. 2004. Characterization of a WRKY transcription factor expressed in late torpedo-stage embryos of Solanum chacoense. Planta 219:185-189. 10.1007/s00425004-1253-2

Lai Z, Vinod K, Zheng Z, Fan B, and Chen Z. 2008. Roles of Arabidopsis WRKY3 and WRKY4 transcription factors in plant responses to pathogens. BMC Plant Biol 8:68. 10.1186/14712229-8-68

Larkin MA, Blackshields G, Brown NP, Chenna R, McGettigan PA, McWilliam H, Valentin F, Wallace IM, Wilm A, Lopez R, Thompson JD, Gibson TJ, and Higgins DG. 2007. Clustal $\mathrm{W}$ and Clustal X version 2.0. Bioinformatics 23:2947-2948. 10.1093/bioinformatics/btm404

Letunic I, Doerks T, and Bork P. 2015. SMART: recent updates, new developments and status in 2015. Nucleic Acids Res 43:D257-260. 10.1093/nar/gku949

Li H, and Durbin R. 2009. Fast and accurate short read alignment with Burrows-Wheeler transform. Bioinformatics 25:1754-1760. 10.1093/bioinformatics/btp324

Li J, Brader G, Kariola T, and Palva ET. 2006. WRKY70 modulates the selection of signaling pathways in plant defense. Plant J 46:477-491. 10.1111/j.1365-313X.2006.02712.x

Ling J, Jiang W, Zhang Y, Yu H, Mao Z, Gu X, Huang S, and Xie B. 2011. Genome-wide analysis of WRKY gene family in Cucumis sativus. BMC Genomics 12:471. 10.1186/1471-2164$12-471$ 
731

732

733

734

735

736

737

738

739

740

741

742

743

744

745

746

747

748

749

750

751

752

753

754

755

756

757

758

759

760

761

762

763

764

765

766

767

768

769
Liu J, Chen X, Liang X, Zhou X, Yang F, Liu J, He SY, and Guo Z. 2016. Alternative splicing of rice WRKY62 and WRKY76 transcription factor genes in pathogen defense. Plant Physiol.

Liu JJ, and Ekramoddoullah AK. 2009. Identification and characterization of the WRKY transcription factor family in Pinus monticola. Genome 52:77-88. 10.1139/G08-106

Maeo K, Hayashi S, Kojima-Suzuki H, Morikami A, and Nakamura K. 2001. Role of conserved residues of the WRKY domain in the DNA-binding of tobacco WRKY family proteins. Biosci Biotechnol Biochem 65:2428-2436. 10.1271/bbb.65.2428

Maher C, Stein L, and Ware D. 2006. Evolution of Arabidopsis microRNA families through duplication events. Genome Res 16:510-519. 10.1101/gr.4680506

Mangelsen E, Kilian J, Berendzen KW, Kolukisaoglu UH, Harter K, Jansson C, and Wanke D. 2008. Phylogenetic and comparative gene expression analysis of barley (Hordeum vulgare) WRKY transcription factor family reveals putatively retained functions between monocots and dicots. BMC Genomics 9:194. 10.1186/1471-2164-9-194

McInerney EM, Rose DW, Flynn SE, Westin S, Mullen TM, Krones A, Inostroza J, Torchia J, Nolte RT, Assa-Munt N, Milburn MV, Glass CK, and Rosenfeld MG. 1998. Determinants of coactivator LXXLL motif specificity in nuclear receptor transcriptional activation. Genes Dev 12:3357-3368. 10.1101/gad.12.21.3357

Murray SL, Ingle RA, Petersen LN, and Denby KJ. 2007. Basal resistance against Pseudomonas syringae in Arabidopsis involves WRKY53 and a protein with homology to a nematode resistance protein. Mol Plant Microbe Interact 20:1431-1438. 10.1094/MPMI-20-11-1431

Muthamilarasan M, Bonthala VS, Khandelwal R, Jaishankar J, Shweta S, Nawaz K, and Prasad M. 2015. Global analysis of WRKY transcription factor superfamily in Setaria identifies potential candidates involved in abiotic stress signaling. Front Plant Sci 6:910. 10.3389/fpls.2015.00910

Ohno S, Wolf U, and Atkin NB. 2009. Evolution from Fish to Mammals by Gene Duplication. Hereditas 59:169-187. 10.1111/j.1601-5223.1968.tb02169.x

Overbeek R, Fonstein M, D'Souza M, Pusch GD, and Maltsev N. 1999. The use of gene clusters to infer functional coupling. Proceedings of the National Academy of Sciences 96:28962901. 10.1073/pnas.96.6.2896

Pleguezuelo CRR, Zuazo VHD, Bielders C, Bocanegra JAJ, Pereatorres F, and Martínez JRF. 2014. Bioenergy farming using woody crops. A review. Agronomy for Sustainable Development 35:95-119.

Pnueli L, Hallak-Herr E, Rozenberg M, Cohen M, Goloubinoff P, Kaplan A, and Mittler R. 2002. Molecular and biochemical mechanisms associated with dormancy and drought tolerance in the desert legume Retama raetam. The Plant Journal 31:319-330. 10.1046/j.1365313X.2002.01364.X

Punta M, Coggill PC, Eberhardt RY, Mistry J, Tate J, Boursnell C, Pang N, Forslund K, Ceric G, Clements J, Heger A, Holm L, Sonnhammer EL, Eddy SR, Bateman A, and Finn RD. 
770

771

772

773

774

775

776

777

778

779

780

781

782

783

784

785

786

787

788

789

790

791

792

793

794

795

796

797

798

799

800

801

802

803

804

805

806

807

808

2012. The Pfam protein families database. Nucleic Acids Res 40:D290-301. 10.1093/nar/gkr1065

Ramamoorthy R, Jiang SY, Kumar N, Venkatesh PN, and Ramachandran S. 2008. A comprehensive transcriptional profiling of the WRKY gene family in rice under various abiotic and phytohormone treatments. Plant Cell Physiol 49:865-879. 10.1093/pcp/pcn061

Rinerson CI, Rabara RC, Tripathi P, Shen QJ, and Rushton PJ. 2015. The evolution of WRKY transcription factors. BMC Plant Biol 15:66. 10.1186/s12870-015-0456-y

Robatzek S, and Somssich IE. 2002. Targets of AtWRKY6 regulation during plant senescence and pathogen defense. Genes Dev 16:1139-1149. 10.1101/gad.222702

Ross CA, Liu Y, and Shen QJ. 2007. The WRKY Gene Family in Rice (Oryza sativa). Journal of Integrative Plant Biology 49:827-842. 10.1111/j.1744-7909.2007.00504.X

Rossberg M, Theres K, Acarkan A, Herrero R, Schmitt T, Schumacher K, Schmitz G, and Schmidt R. 2001. Comparative Sequence Analysis Reveals Extensive Microcolinearity in the Lateral Suppressor Regions of the Tomato, Arabidopsis, and Capsella Genomes. Plant Cell 13:979. 10.2307/3871354

Rushton PJ, Somssich IE, Ringler P, and Shen QJ. 2010. WRKY transcription factors. Trends Plant Sci 15:247-258. 10.1016/j.tplants.2010.02.006

Sun C. 2003. A Novel WRKY Transcription Factor, SUSIBA2, Participates in Sugar Signaling in Barley by Binding to the Sugar-Responsive Elements of the iso1 Promoter. The Plant Cell Online 15:2076-2092. 10.1105/tpc.014597

Suyama M, Torrents D, and Bork P. 2006. PAL2NAL: robust conversion of protein sequence alignments into the corresponding codon alignments. Nucleic Acids Res 34:W609-612. 10.1093/nar/gkl315

Tamura K, Stecher G, Peterson D, Filipski A, and Kumar S. 2013. MEGA6: Molecular Evolutionary Genetics Analysis version 6.0. Molecular Biology and Evolution 30:27252729. 10.1093/molbev/mst197

Ulker B, and Somssich IE. 2004. WRKY transcription factors: from DNA binding towards biological function. Curr Opin Plant Biol 7:491-498. 10.1016/j.pbi.2004.07.012

van Verk MC, Pappaioannou D, Neeleman L, Bol JF, and Linthorst HJ. 2008. A Novel WRKY transcription factor is required for induction of PR-1a gene expression by salicylic acid and bacterial elicitors. Plant Physiol 146:1983-1995. 10.1104/pp.107.112789

Vision TJ, Brown DG, and Tanksley SD. 2000. The Origins of Genomic Duplications in Arabidopsis. Science 290:2114-2117. 10.1126/science.290.5499.2114

Wagner GP, Kin K, and Lynch VJ. 2012. Measurement of mRNA abundance using RNA-seq data: RPKM measure is inconsistent among samples. Theory Biosci 131:281-285. 10.1007/s12064-012-0162-3

Wang Y, Feng L, Zhu Y, Li Y, Yan H, and Xiang Y. 2015. Comparative genomic analysis of the WRKY III gene family in populus, grape, arabidopsis and rice. Biology direct 10:1. 
809

810

811

812

813

814

815

816

817

818

819

820

821

822

823

824

825

826

827

828

829

830

831

832

833

834

835

836

837

838

839

840

841

842

843

844

845

846

847

Wei W, Zhang Y, Han L, Guan Z, and Chai T. 2008. A novel WRKY transcriptional factor from Thlaspi caerulescens negatively regulates the osmotic stress tolerance of transgenic tobacco. Plant Cell Rep 27:795-803.

Wu KL. 2005. The WRKY Family of Transcription Factors in Rice and Arabidopsis and Their Origins. DNA Research 12:9-26. 10.1093/dnares/12.1.9

Xie Z, Zhang ZL, Zou X, Huang J, Ruas P, Thompson D, and Shen QJ. 2005. Annotations and functional analyses of the rice WRKY gene superfamily reveal positive and negative regulators of abscisic acid signaling in aleurone cells. Plant Physiol 137:176-189. 10.1104/pp.104.054312

Xiong W, Xu X, Zhang L, Wu P, Chen Y, Li M, Jiang H, and Wu G. 2013. Genome-wide analysis of the WRKY gene family in physic nut (Jatropha curcas L.). Gene 524:124-132. 10.1016/j.gene.2013.04.047

Xu X, Chen C, Fan B, and Chen Z. 2006. Physical and functional interactions between pathogeninduced Arabidopsis WRKY18, WRKY40, and WRKY60 transcription factors. Plant Cell 18:1310-1326. 10.1105/tpc.105.037523

Yamasaki K, Kigawa T, Inoue M, Tateno M, Yamasaki T, Yabuki T, Aoki M, Seki E, Matsuda T, Tomo Y, Hayami N, Terada T, Shirouzu M, Tanaka A, Seki M, Shinozaki K, and Yokoyama S. 2005. Solution structure of an Arabidopsis WRKY DNA binding domain. Plant Cell 17:944-956. 10.1105/tpc.104.026435

Yang Z. 2007. PAML 4: phylogenetic analysis by maximum likelihood. Molecular Biology and Evolution 24:1586-1591. 10.1093/molbev/msm088

Zhang J. 2003. Evolution by gene duplication: an update. Trends in Ecology \& Evolution 18:292298. 10.1016/s0169-5347(03)00033-8

Zhang Y, and Wang L. 2005. The WRKY transcription factor superfamily: its origin in eukaryotes and expansion in plants. BMC Evol Biol 5:1. 10.1186/1471-2148-5-1

Zhang ZL, Xie Z, Zou X, Casaretto J, Ho TH, and Shen QJ. 2004. A rice WRKY gene encodes a transcriptional repressor of the gibberellin signaling pathway in aleurone cells. Plant Physiol 134:1500-1513. 10.1104/pp.103.034967

Zhou QY, Tian AG, Zou HF, Xie ZM, Lei G, Huang J, Wang CM, Wang HW, Zhang JS, and Chen SY. 2008. Soybean WRKY-type transcription factor genes, GmWRKY13, GmWRKY21, and GmWRKY54, confer differential tolerance to abiotic stresses in transgenic Arabidopsis plants. Plant Biotechnol J 6:486-503. 10.1111/j.14677652.2008.00336.x

Zou X, Seemann JR, Neuman D, and Shen QJ. 2004. A WRKY gene from creosote bush encodes an activator of the abscisic acid signaling pathway. J Biol Chem 279:55770-55779. 10.1074/jbc.M408536200

Zou Z, Yang L, Wang D, Huang Q, Mo Y, and Xie G. 2016. Gene Structures, Evolution and Transcriptional Profiling of the WRKY Gene Family in Castor Bean (Ricinus communis L.). PLoS One 11:e0148243. 10.1371/journal.pone.0148243 


\section{Figure 1}

Comparison of the WRKY domain sequences from 85 SsWRKY genes.

The WRKY gene with the suffix $-\mathrm{N}$ and $-\mathrm{C}$ indicates the $\mathrm{N}$-terminal and $\mathrm{C}$-terminal WRKY domain of group I members, respectively. "-" has been inserted for the optimal alignment. Red indicates the highly conserved WRKYGQK heptapeptide, and the zinc finger motifs are highlighted in green. The position of a conserved intron is indicated by an arrowhead. 


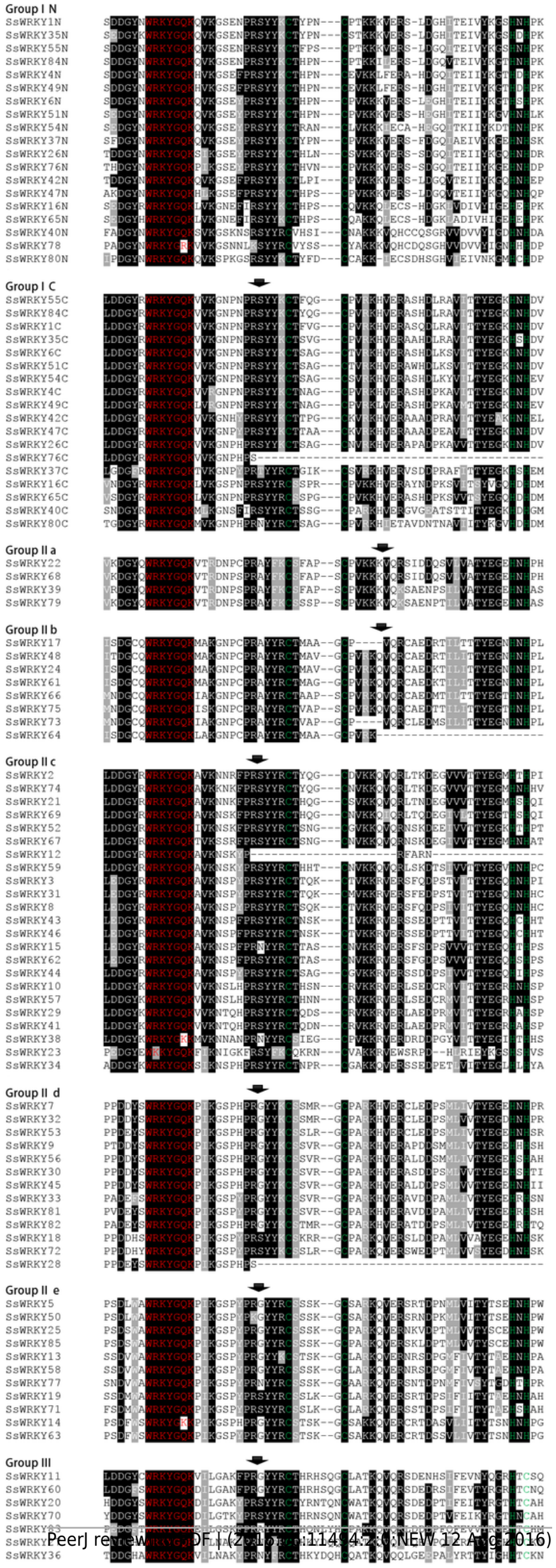




\section{Figure 2}

Chromosomal location of SsWRKY genes.

Red indicates group I, blue indicates group II and green indicates group III. Red lines indicate gene clusters. The chromosome numbers are given at the top of each chromosome and the left side of each chromosome is related to the approximate physical location of each WRKY gene. Only one unmapped SsWRKY gene is shown on ChrN. 


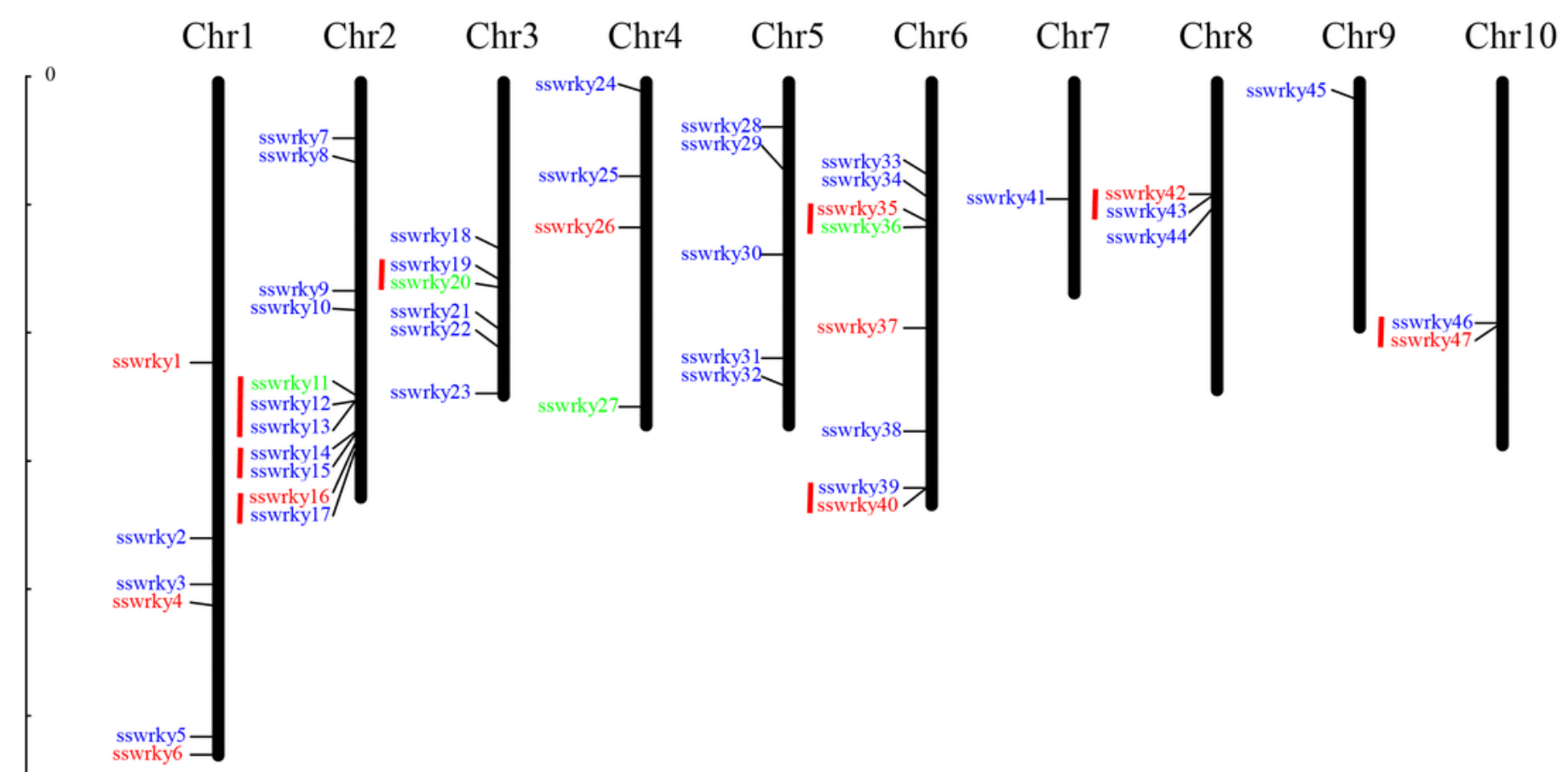

$30 \mathrm{Mb}$

Chr11 Chr12 Chr13 Chr14 Chr15 Chr16 Chr17 Chr18 Chr19 ChrN
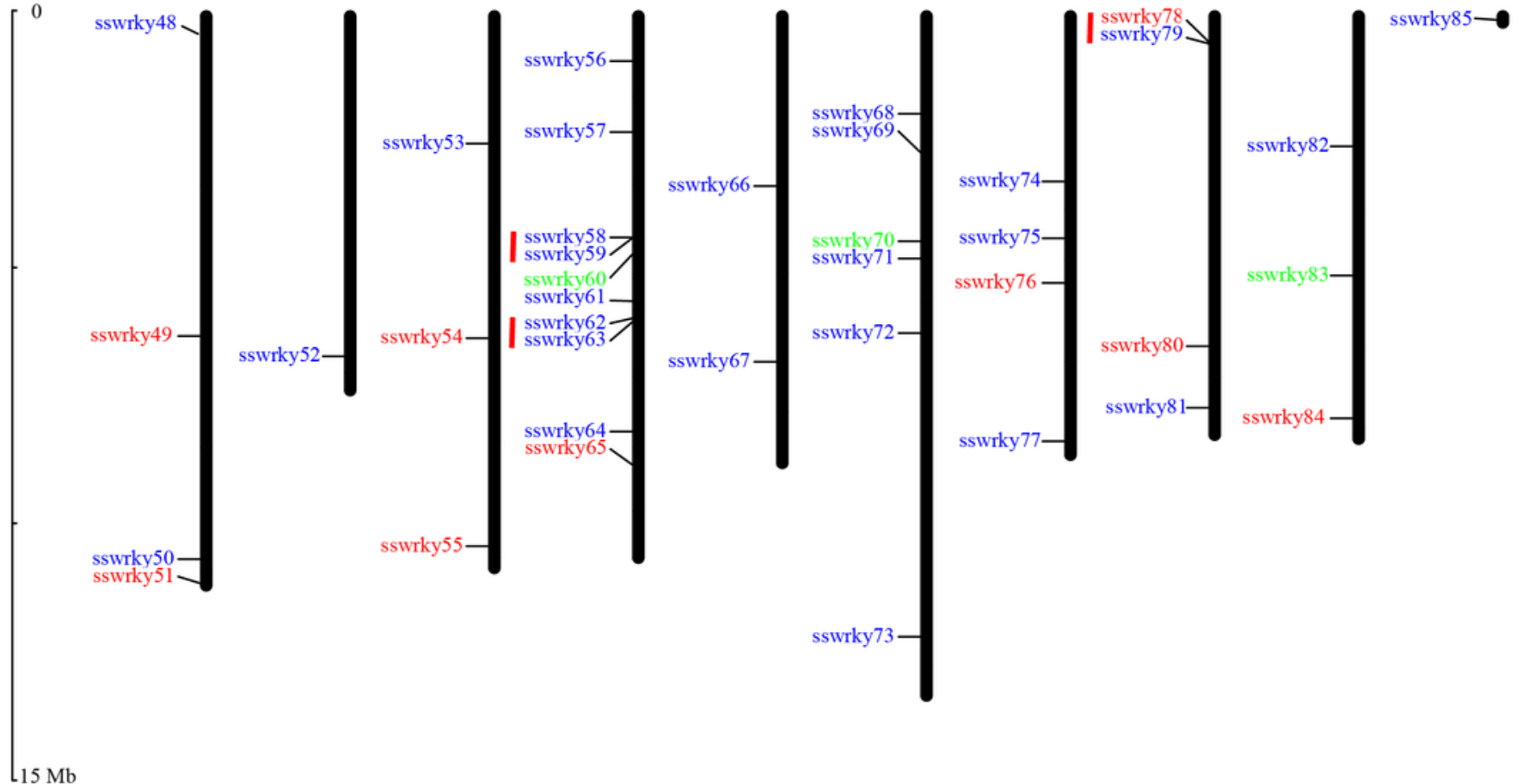


\section{Figure 3}

Phylogenetic tree of WRKY domains from willow and Arabidopsis.

The phylogenetic tree was constructed using the neighbor-joining method in MEGA 6.0. The WRKY genes with the suffix ' $\mathrm{N}$ ' and ' $\mathrm{C}$ ' indicate the $\mathrm{N}$-terminal and the C-terminal WRKY domains of group I, respectively. The different colors indicate different groups (I, II and III) or subgroups (Ila, b, c, d and e) of WRKY domains. Circles indicate WRKY genes from willow, and diamonds represent genes from Arabidopsis. The purple trapezoid region indicate a new subgroup belonging to Ilc. 


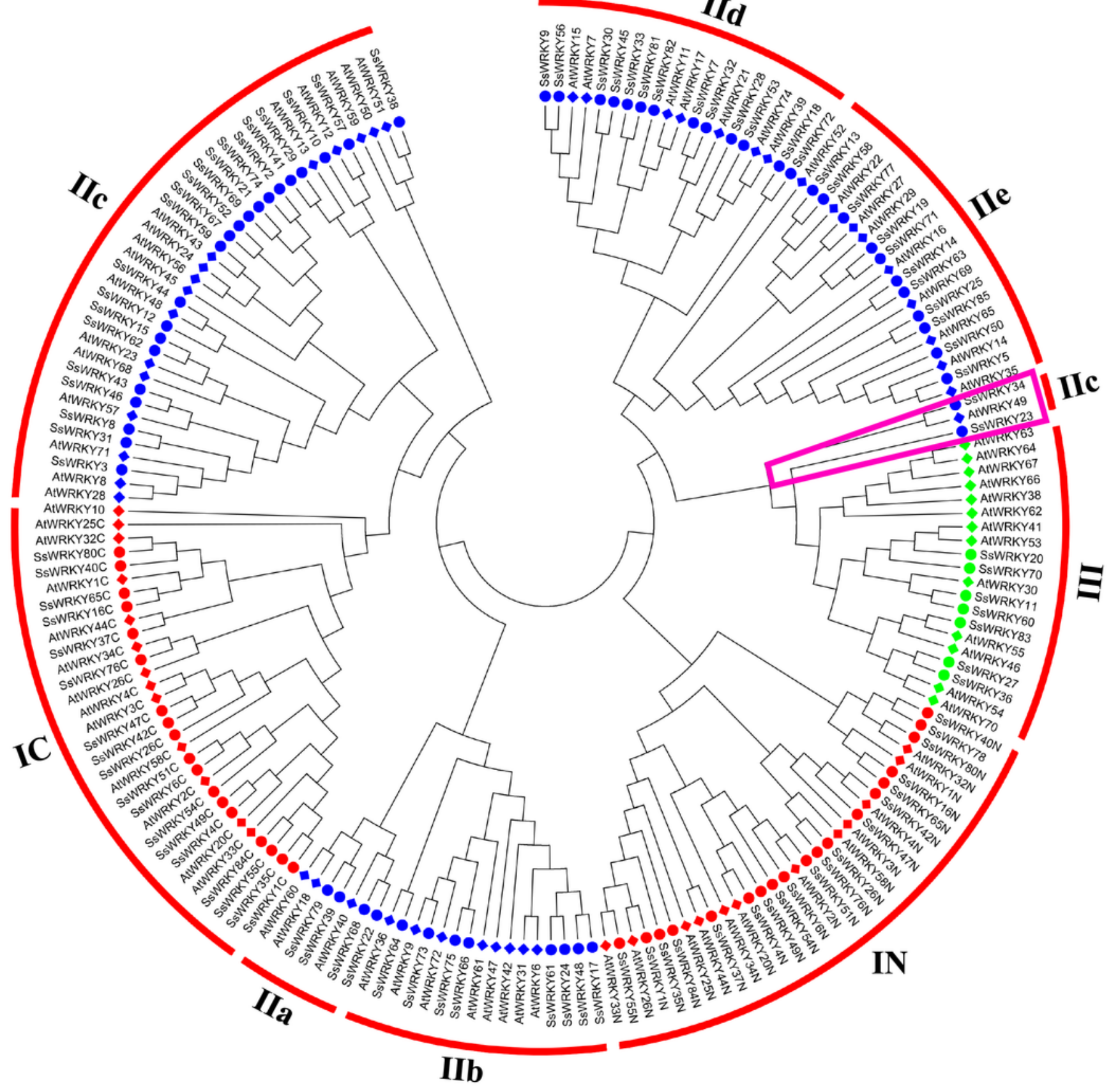




\section{Figure 4}

Genomic organization of SsWRKY genes.

A) The phylogenetic tree built on the basis of full-length SsWRKY genes was depicted using the neighbor-joining method in MEGA 6.0. The short black lines indicate the existence of duplicated gene pairs; B) The graphic exon-intron structure of SsWRKY genes is displayed using GSDS. Green indicates exons, and gray indicates introns. The introns phases 0,1 and 2 are indicated by numbers 0,1 and 2, respectively. 
A

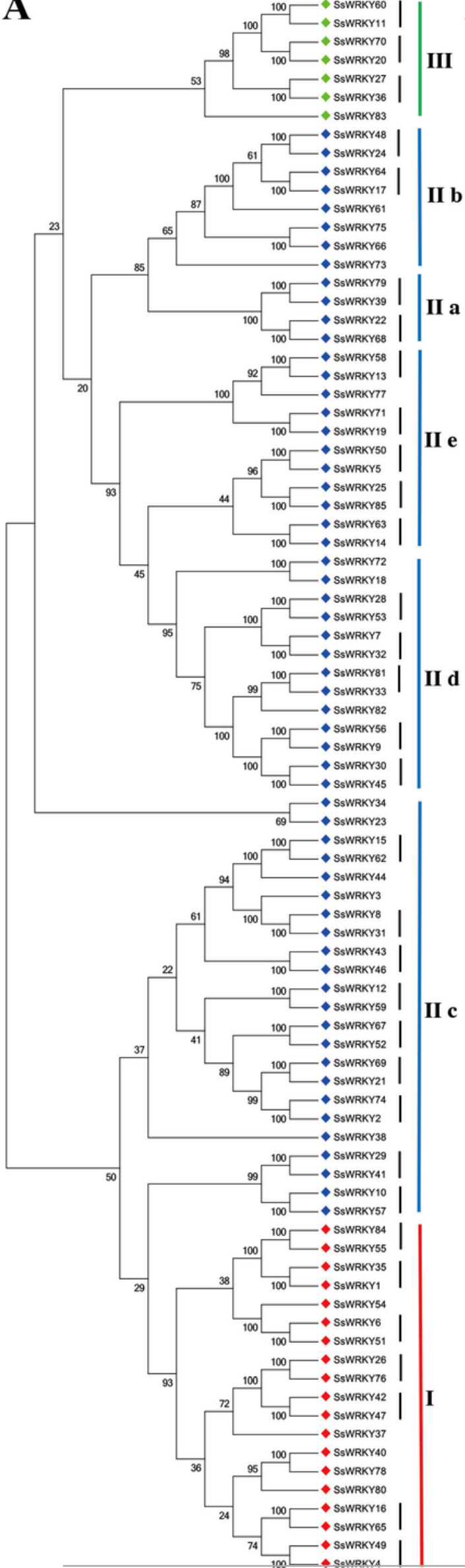

B

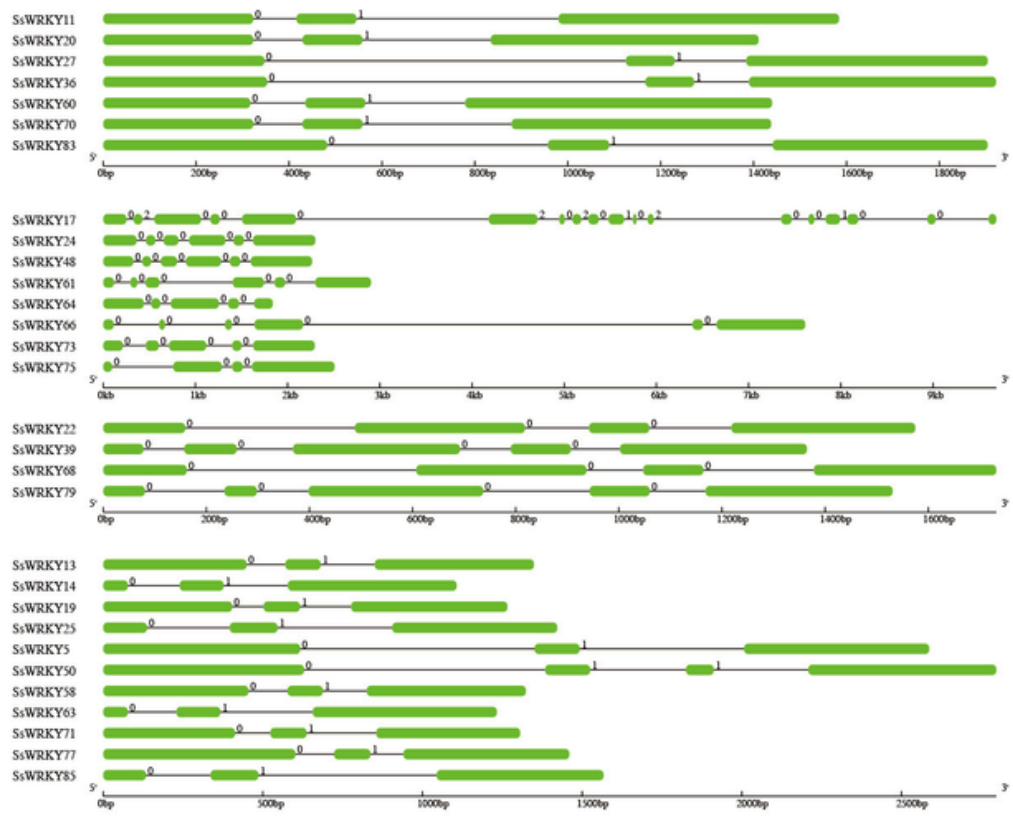

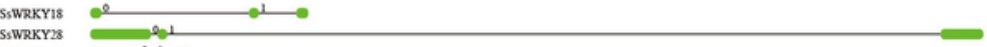

SsWRKY30

SSWRKY3 2

SsWRKY33 to 2010

SsWRKY45

StWRKYST

SsWRKYSO

$2+1$

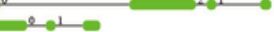

SsWRKYsI $=2-10$

SSWRKYY 2

SswRKY
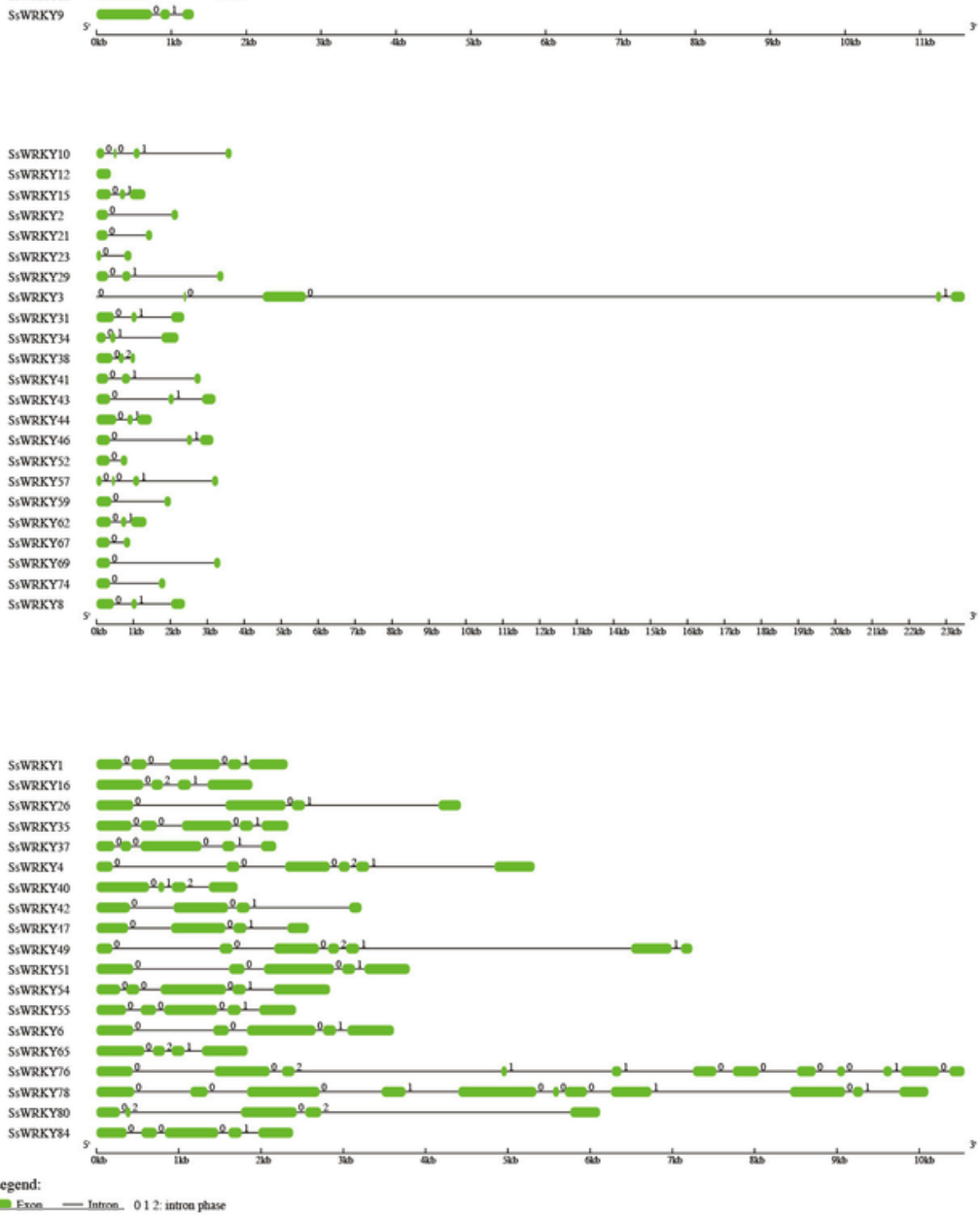

Legend: 


\section{Figure 5}

Expression profiles of the 85 SsWRKY genes in root, stem, bark, bud and leaf.

Color scale represents RPKM normalized log2 transformed counts and red indicates high expression, blue indicates low expression and white indicates the gene is not expressed in this tissue. 


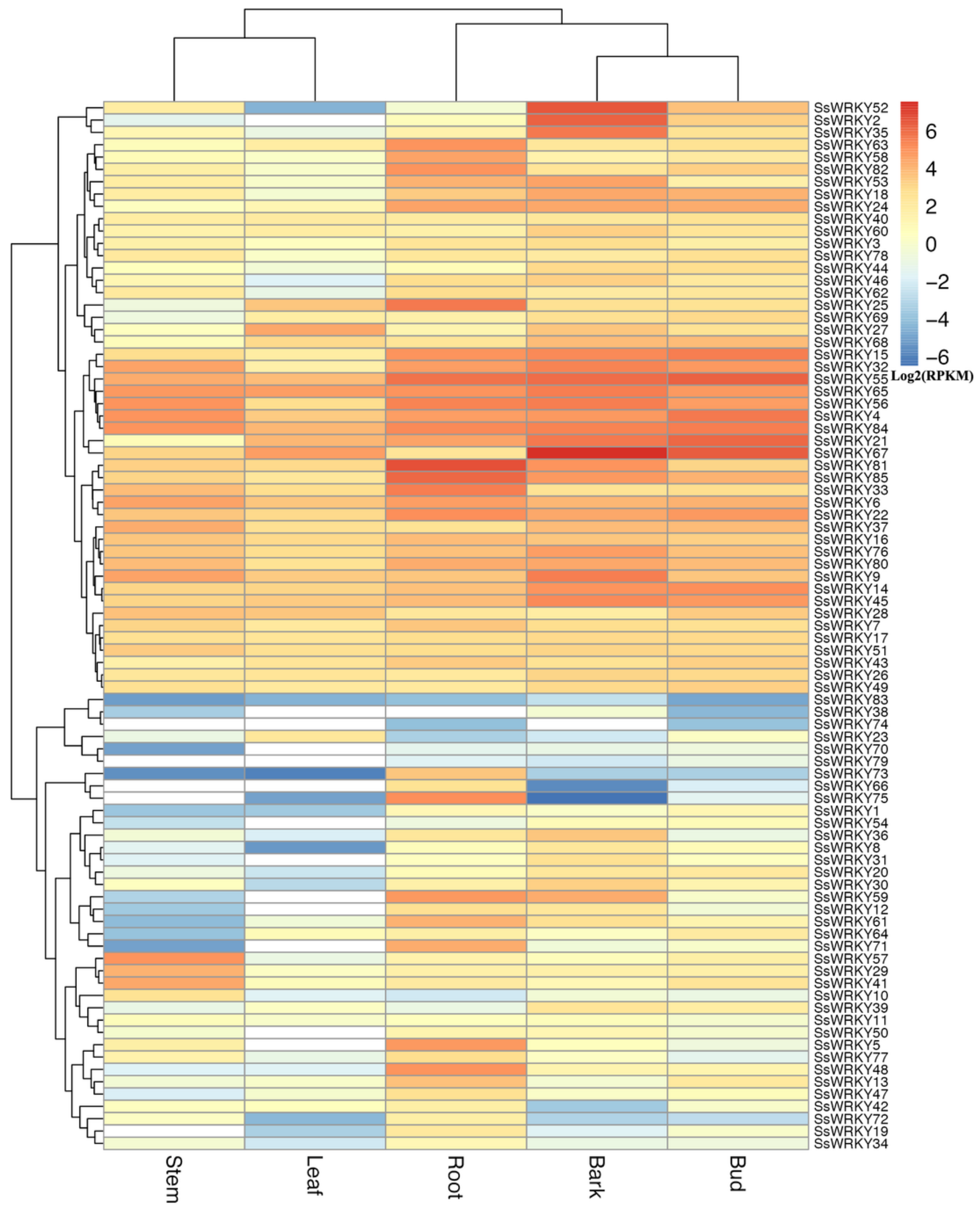




\section{Table $\mathbf{1}$ (on next page)}

The detailed characteristics of WRKY genes identified in willow. 
1 Table 1. The detailed characteristics of WRKY genes identified in willow.

\begin{tabular}{|c|c|c|c|c|c|c|c|c|c|}
\hline \multirow[t]{2}{*}{ Gene } & \multirow[t]{2}{*}{ SequenceID } & \multirow[t]{2}{*}{ Chr } & \multirow[t]{2}{*}{ Group } & \multicolumn{2}{|l|}{ Ortholog } & \multicolumn{3}{|c|}{ Deduced polypeptide } & \multirow[t]{2}{*}{ Introns } \\
\hline & & & & AtWRKY & PtWRKY & Length(aa) & PI & MW(kDa) & \\
\hline SsWRKY1 & willow_GLEAN_10011238 & 1 & I & 33 & 17 & 583 & 7.14 & 64.7 & 4 \\
\hline SsWRKY2 & willow_GLEAN_10019192 & 1 & II c & 45 & 43 & 162 & 9.47 & 18.6 & 1 \\
\hline SsWRKY3 & willow_GLEAN_10017208 & 1 & II c & 28,71 & 29 & 584 & 9.42 & 65.6 & 4 \\
\hline SsWRKY4 & willow_GLEAN_10017139 & 1 & I & 20 & 44 & 560 & 6.99 & 60.9 & 5 \\
\hline SsWRKY5 & willow_GLEAN_10007860 & 1 & II e & 35 & 45 & 445 & 5.92 & 48.4 & 2 \\
\hline SsWRKY6 & willow_GLEAN_10003806 & 1 & I & 2 & $37,101,102$ & 733 & 5.69 & 78.8 & 4 \\
\hline SsWRKY7 & willow_GLEAN_10022392 & 2 & II d & 21 & 46,63 & 453 & 9.53 & 49.9 & 4 \\
\hline SsWRKY8 & willow_GLEAN_10022273 & 2 & II c & 71 & 47 & 328 & 6.89 & 37.0 & 2 \\
\hline SsWRKY9 & willow_GLEAN_10009329 & 2 & II d & 15 & 14,94 & 339 & 9.77 & 37.5 & 2 \\
\hline SsWRKY10 & willow_GLEAN_10009231 & 2 & II c & 12 & 48 & 204 & 7.64 & 23.6 & 3 \\
\hline SsWRKY11 & willow_GLEAN_10016913 & 2 & III & 30 & 6,51 & 351 & 6.27 & 39.2 & 2 \\
\hline SsWRKY12 & willow_GLEAN_10016886 & 2 & II c & - & 19,50 & 129 & 6.75 & 14.6 & 0 \\
\hline SsWRKY13 & willow_GLEAN_10016883 & 2 & II e & 22 & $23,49,78$ & 352 & 5.81 & 38.3 & 2 \\
\hline SsWRKY14 & willow_GLEAN_10019911 & 2 & II e & - & 3 & 247 & 5.58 & 28.1 & 2 \\
\hline SsWRKY15 & willow_GLEAN_10019925 & 2 & II c & 23 & 13,33 & 319 & 6.46 & 35.6 & 2 \\
\hline SsWRKY16 & willow_GLEAN_10019982 & 2 & I & 1 & 54 & 472 & 6.88 & 52.2 & 3 \\
\hline SsWRKY17 & willow_GLEAN_10020022 & 2 & II $\mathrm{b}$ & 47 & 53 & 1081 & 5.25 & 116.8 & 17 \\
\hline SsWRKY18 & willow_GLEAN_10025583 & 3 & II d & - & 55 & 142 & 9.60 & 16.5 & 2 \\
\hline SsWRKY19 & willow_GLEAN_10025423 & 3 & II e & 29 & 41 & 335 & 5.54 & 37.9 & 2 \\
\hline SsWRKY20 & willow_GLEAN_10025378 & 3 & III & $41 / 53$ & 21 & 342 & 5.25 & 38.4 & 2 \\
\hline SsWRKY21 & willow_GLEAN_10008020 & 3 & $\Pi \mathrm{c}$ & 45 & 18 & 157 & 9.41 & 17.8 & 1 \\
\hline SsWRKY22 & willow_GLEAN_10006448 & 3 & II a & 40 & 88 & 320 & 8.38 & 35.4 & 3 \\
\hline SsWRKY23 & willow_GLEAN_10013342 & 3 & II c & - & 39 & 109 & 8.03 & 12.9 & 1 \\
\hline SsWRKY24 & willow_GLEAN_10009960 & 4 & II $\mathrm{b}$ & 42 & 28,79 & 604 & 6.93 & 65.3 & 5 \\
\hline SsWRKY25 & willow_GLEAN_10017267 & 4 & II e & 65 & 8,58 & 267 & 5.43 & 29.7 & 2 \\
\hline SsWRKY26 & willow_GLEAN_10018559 & 4 & I & 58 & 60 & 537 & 8.72 & 58.9 & 3 \\
\hline SsWRKY27 & willow_GLEAN_10004854 & 4 & III & 54 & 85 & 323 & 5.70 & 36.3 & 2 \\
\hline SsWRKY28 & willow_GLEAN_10008312 & 5 & II d & - & - & 490 & 10.27 & 54.0 & 2 \\
\hline SsWRKY29 & willow_GLEAN_10009112 & 5 & II c & 13 & 68 & 235 & 8.70 & 26.7 & 2 \\
\hline SsWRKY30 & willow_GLEAN_10003565 & 5 & II d & 15 & 20 & 310 & 9.48 & 34.3 & 2 \\
\hline SsWRKY31 & willow_GLEAN_10016009 & 5 & $\Pi \mathrm{c}$ & 28,71 & 62 & 322 & 6.67 & 36.2 & 2 \\
\hline SsWRKY32 & willow_GLEAN_10018195 & 5 & II d & 21 & 46,63 & 349 & 9.69 & 38.8 & 2 \\
\hline SsWRKY33 & willow_GLEAN_10026833 & 6 & II d & 7 & 91 & 339 & 9.89 & 36.8 & 3 \\
\hline SsWRKY34 & willow_GLEAN_10026721 & 6 & II c & 49 & 34 & 287 & 5.25 & 32.1 & 2 \\
\hline SsWRKY35 & willow_GLEAN_10026591 & 6 & I & 33 & 64 & 572 & 6.41 & 62.7 & 4 \\
\hline SsWRKY36 & willow_GLEAN_10026566 & 6 & III & 54 & 85 & 329 & 6.13 & 36.7 & 2 \\
\hline
\end{tabular}




\begin{tabular}{|c|c|c|c|c|c|c|c|c|}
\hline SsWRKY37 & willow_GLEAN_10020588 & 6 & I & 44 & 93 & 478 & 9.25 & 52.5 \\
\hline SsWRKY38 & willow_GLEAN_10026166 & 6 & II $\mathrm{c}$ & 51 & 67 & 233 & 5.03 & 26.1 \\
\hline SsWRKY39 & willow_GLEAN_10026455 & 6 & II a & $18 / 60$ & 9 & 327 & 9.02 & 36.2 \\
\hline SsWRKY40 & willow_GLEAN_10026458 & 6 & I & 32 & 15 & 413 & 8.26 & 44.9 \\
\hline SsWRKY41 & willow_GLEAN_10008192 & 7 & I $\mathrm{c}$ & 13 & 68 & 236 & 9.21 & 26.6 \\
\hline SsWRKY42 & willow_GLEAN_10025108 & 8 & I & $3 / 4$ & 69 & 460 & 8.80 & 50.6 \\
\hline SsWRKY43 & willow_GLEAN_10025123 & 8 & II $\mathrm{c}$ & 57 & 71 & 295 & 6.32 & 32.3 \\
\hline SsWRKY44 & willow_GLEAN_10015641 & 8 & II $\mathrm{c}$ & 48 & 70 & 357 & 6.11 & 39.9 \\
\hline SsWRKY45 & willow_GLEAN_10008155 & 9 & II d & 15 & 20,26 & 331 & 9.57 & 36.4 \\
\hline SsWRKY46 & willow_GLEAN_10013562 & 10 & II $\mathrm{c}$ & 57 & 71 & 289 & 6.26 & 31.9 \\
\hline SsWRKY47 & willow_GLEAN_10013586 & 10 & I & $3 / 4$ & 72 & 490 & 8.60 & 53.7 \\
\hline SsWRKY48 & willow_GLEAN_10004012 & 11 & II $\mathrm{b}$ & 42 & 100 & 585 & 6.48 & 63.3 \\
\hline SsWRKY49 & willow_GLEAN_10006060 & 11 & I & 20 & 44 & 607 & 7.09 & 6.6 \\
\hline SsWRKY50 & willow_GLEAN_10007614 & 11 & I $\mathrm{e}$ & 35 & 74 & 481 & 5.39 & 51.6 \\
\hline SsWRKY51 & willow_GLEAN_10007542 & 11 & I & 2 & 37 & 734 & 6.10 & 79.7 \\
\hline SsWRKY52 & willow_GLEAN_10013801 & 12 & II $\mathrm{c}$ & - & 75 & 178 & 9.08 & 20.5 \\
\hline SsWRKY53 & willow_GLEAN_10012158 & 13 & II d & 74 & 25 & 356 & 9.66 & 40.0 \\
\hline SsWRKY54 & willow_GLEAN_10004417 & 13 & I & 2 & 35 & 697 & 6.52 & 76.1 \\
\hline SsWRKY55 & willow_GLEAN_10007732 & 13 & I & 33 & 1 & 602 & 7.65 & 66.0 \\
\hline SsWRKY56 & willow_GLEAN_10009039 & 14 & II d & 15 & 14,94 & 362 & 9.39 & 40.0 \\
\hline SsWRKY57 & willow_GLEAN_10016668 & 14 & II $\mathrm{c}$ & 12 & 48 & 180 & 8.47 & 20.7 \\
\hline SsWRKY58 & willow_GLEAN_10016177 & 14 & II e & 22 & $23,49,78$ & 354 & 6.35 & 38.8 \\
\hline SsWRKY59 & willow_GLEAN_10016180 & 14 & II c & 43 & 19,50 & 193 & 9.47 & 21.7 \\
\hline SsWRKY60 & willow_GLEAN_10016220 & 14 & III & 30 & 6 & 368 & 5.03 & 41.3 \\
\hline SsWRKY61 & willow_GLEAN_10018940 & 14 & II $\mathrm{b}$ & 42 & 28,79 & 467 & 8.78 & 50.0 \\
\hline SsWRKY62 & willow_GLEAN_10018891 & 14 & II $\mathrm{c}$ & 23 & 13,33 & 318 & 5.71 & 35.6 \\
\hline SsWRKY63 & willow_GLEAN_10018881 & 14 & II e & - & 80 & 263 & 5.05 & 29.7 \\
\hline SsWRKY64 & willow_GLEAN_10020302 & 14 & I $\mathrm{b}$ & 36 & - & 460 & 6.28 & 50.0 \\
\hline SsWRKY65 & willow_GLEAN_10020380 & 14 & I & 1 & 2 & 481 & 5.98 & 52.8 \\
\hline SsWRKY66 & willow_GLEAN_10011119 & 15 & II $\mathrm{b}$ & 9 & 99 & 618 & 6.55 & 66.2 \\
\hline SsWRKY67 & willow_GLEAN_10016438 & 15 & $\Pi \mathrm{c}$ & - & 82 & 178 & 9.35 & 20.5 \\
\hline SsWRKY68 & willow_GLEAN_10023347 & 16 & I $\mathrm{a}$ & 40 & 88 & 320 & 8.82 & 35.3 \\
\hline SsWRKY69 & willow_GLEAN_10023447 & 16 & II $\mathrm{c}$ & 45 & 18 & 178 & 9.17 & 20.1 \\
\hline SsWRKY70 & willow_GLEAN_10023687 & 16 & III & $41 / 53$ & 21 & 336 & 5.17 & 37.2 \\
\hline SsWRKY71 & willow_GLEAN_10023735 & 16 & II e & 29 & 41 & 325 & 5.54 & 36.6 \\
\hline SsWRKY72 & willow_GLEAN_10014752 & 16 & II d & - & 55 & 338 & 9.24 & 37.9 \\
\hline SsWRKY73 & willow_GLEAN_10009602 & 16 & I $\mathrm{b}$ & 9 & 42 & 509 & 5.51 & 55.3 \\
\hline SsWRKY74 & willow_GLEAN_10010473 & 17 & II $\mathrm{c}$ & 45 & 43 & 182 & 9.92 & 20.9 \\
\hline SsWRKY75 & willow_GLEAN_10015128 & 17 & II $\mathrm{b}$ & 9 & 86 & 544 & 6.01 & 59.0 \\
\hline
\end{tabular}




$\begin{array}{llllllllll}\text { SsWRKY76 } & \text { willow_GLEAN_10015184 } & 17 & \text { I } & 58 & 87 & 1044 & 8.94 & 116.1 \\ \text { SsWRKY77 } & \text { willow_GLEAN_10005468 } & 17 & \text { II e } & 27 & 96 & 411 & 5.96 & 45.7 & 2 \\ \text { SsWRKY78 } & \text { willow_GLEAN_10006860 } & 18 & \text { I } & - & 90 & 1593 & 8.67 & 179.0 & 10 \\ \text { SsWRKY79 } & \text { willow_GLEAN_10006862 } & 18 & \text { II a } & 18 / 60 & 9 & 320 & 8.57 & 35.6 & 4 \\ \text { SsWRKY80 } & \text { willow_GLEAN_10011608 } & 18 & \text { I } & 32 & - & 528 & 5.74 & 57.8 & 4 \\ \text { SsWRKY81 } & \text { willow_GLEAN_10004546 } & 18 & \text { II d } & 7 & 7,91 & 300 & 9.80 & 32.8 & 2 \\ \text { SsWRKY82 } & \text { willow_GLEAN_10003422 } & 19 & \text { II d } & 11 / 17 & 24 & 339 & 9.58 & 37.1 & 2 \\ \text { SsWRKY83 } & \text { willow_GLEAN_10011321 } & 19 & \text { II } & 55 & 36,76 & 358 & 5.63 & 38.7 & 2 \\ \text { SsWRKY84 } & \text { willow_GLEAN_10005288 } & 19 & \text { I } & 33 & 4 & 597 & 6.69 & 65.6 & 4 \\ \text { SsWRKY85 } & \text { willow_GLEAN_10002834 } & \text { N/A } & \text { II e } & 65 & 58 & 268 & 5.83 & 30.2\end{array}$

2 Chr, chromosome numbers.

3 N/A, not available.

4 "-", not detected.

5 